\title{
Überzeugungen und Selbstwirksamkeitserwartungen im Umgang mit kultureller Diversität in ihrem Zusammenspiel mit sprachpädagogischen Förderstrategien
}

\author{
Csaba Kurucz (D) A Axinja Hachfeld • Imke Groeneveld • \\ Hans-Günther Roßbach • Yvonne Anders
}

Online publiziert: 15 . Juli 2020

(C) Der/die Autor(en) 2020

Zusammenfassung Die vorliegende Studie untersucht die Überzeugungen und die Selbstwirksamkeitserwartungen in Bezug auf kulturelle Diversität von frühpädagogischen Fachkräften. Es wurde (1) untersucht, inwieweit multikulturelle Überzeugungen und diversitätsspezifische Selbstwirksamkeitserwartungen miteinander in Beziehung stehen und sich im zeitlichen Verlauf verändern, und (2) in welchem Zusammenhang die beiden Konstrukte mit der wahrgenommenen Wichtigkeit verschiedener sprachpädagogischer Förderstrategien in Kindertageseinrichtungen stehen. Dazu wurden in einer Längsschnittstudie 251 frühpädagogische Fachkräfte

Zusatzmaterial online Zusätzliche Informationen sind in der Online-Version dieses Artikels (https:// doi.org/10.1007/s11618-020-00954-w) enthalten.

Dipl. Soz. C. Kurucz, M.A. $(\bowtie)$

Arbeitsbereich Frühkindliche Bildung und Erziehung, Fachbereich Erziehungswissenschaft und Psychologie, Freie Universität Berlin, Habelschwerdter Allee 45, 14195 Berlin, Deutschland

E-Mail: csaba.kurucz@fu-berlin.de

Prof. Dr. A. Hachfeld

Unterrichtsforschung mit Schwerpunkt Heterogenität, Fachbereich Geschichte und Soziologie mit Empirischer Bildungsforschung, Universität Konstanz, Universitätsstraße 10, 78464 Konstanz, Deutschland

E-Mail: axinja.hachfeld@uni-konstanz.de

Dr. I. Groeneveld

Berlin, Deutschland

E-Mail: imke.groeneveld@gmail.com

Prof. Dr. H.-G. Roßbach

Elementar- und Familienpädagogik, Institut für Erziehungswissenschaft, Universität Bamberg, An der Weberei 5, 96047 Bamberg, Deutschland

E-Mail: hans-guenther.rossbach@uni-bamberg.de

Prof. Dr. Y. Anders

Elementar- und Familienpädagogik, Institut für Erziehungswissenschaft, Universität Bamberg,

Markusstraße 8a, 96045 Bamberg, Deutschland

E-Mail: yvonne.anders@uni-bamberg.de 
mit spezieller sprachpädagogischer Qualifikation zu zwei Messzeitpunkten befragt. Die Ergebnisse des Strukturgleichungsmodells zeigten, dass multikulturelle Überzeugungen und diversitätsspezifische Selbstwirksamkeitserwartungen in Zusammenhang stehende, aber eigenständige Konstrukte sind, die auf unterschiedliche Weise mit der eingeschätzten Wichtigkeit alltagsintegrierter und additiver Sprachförderansätze in kulturell diversen Gruppen assoziiert sind. So konnte für die multikulturellen Überzeugungen - im Vergleich zu den diversitätsspezifischen Selbstwirksamkeitserwartungen - ein signifikanter Zusammenhang zu der intendierten alltagsintegrierten sprachlichen Förderung festgestellt werden. Die Ergebnisse werden abschließend vor dem Hintergrund ihrer Relevanz für zukünftige Forschung und die Ausbildung frühpädagogischer Fachkräfte diskutiert.

Schlüsselwörter Kulturelle Überzeugungen · Diversität · Sprachförderung · Kindergarten $\cdot$ Selbstwirksamkeitserwartungen

\title{
Beliefs and self-efficacy in dealing with cultural diversity in interaction with language support strategies
}

\begin{abstract}
This study addresses cultural beliefs and diversity-specific self-efficacy of early childhood educators. The research questions focused on (1) the extent multicultural beliefs and diversity-specific self-efficacy are interrelated and change over time, and (2) whether the two constructs are differentially related to the perceived importance of different strategies of language and literacy support in day-care centers. In a longitudinal study, 251 early childhood education professionals with special language qualifications filled out questionnaires at two measurement time points. The results from the structural equation models showed that multicultural beliefs and diversity-specific self-efficacy are related but independent constructs that are differentially related to the perceived importance of everyday and additive language opportunities for culturally diverse groups. In contrast to diversity-specific self-efficacy, multicultural beliefs predicted the perceived importance of language education embedded in daily routines. The results are discussed against the background of their relevance to future research and the education of early childhood educators.
\end{abstract}

Keywords Beliefs $\cdot$ Diversity $\cdot$ Language support $\cdot$ Preschool $\cdot$ Self-efficacy

\section{Einleitung}

Kindertageseinrichtungen stehen gegenwärtig vor vielfältigen gesellschaftspolitischen Herausforderungen. So wird einerseits in politischen Diskursen verstärkt der Beitrag frühkindlicher Bildung und Erziehung zur Herstellung von mehr Chancengleichheit betont (Bundesministerium für Familie, Senioren, Frauen und Jugend (BMFSFJ) 2005; BMFSFJ 2008; Brandes et al. 2011), andererseits sieht sich das deutsche Erziehungs- und Bildungssystem nicht erst durch die jüngste Zunahme von Kindern mit Fluchthintergrund mit Herausforderungen aufgrund kultureller Diversität konfrontiert: In der Altersgruppe der Kinder unter sechs Jahren weisen in- 
zwischen $38 \%$ einen Migrationshintergrund ${ }^{1}$ auf (Autorengruppe Bildungsberichterstattung 2018). Die Schullaufbahn von Kindern mit Migrationshintergrund verläuft in Deutschland häufig noch weniger erfolgreich als von Kindern ohne Migrationshintergrund (OECD 2016; Autorengruppe Bildungsberichterstattung 2018). Verschiedene Studien sehen die Ursache dafür in den weniger weit entwickelten Deutschsprachkenntnissen (Schwippert et al. 2012; Kempert et al. 2016). Disparitäten beim Sprachstand zwischen Kindern mit und ohne Migrationshintergrund lassen sich bereits vor Eintritt in Kindertageseinrichtungen feststellen und sind auch zu Beginn der Grundschulzeit weiter evident (Ebert et al. 2013; Relikowski et al. 2015; Linberg und Wenz 2017). Durch eine frühe Sprachförderung im Deutschen könnte Bildungsbenachteiligungen erfolgreich entgegengewirkt werden.

Für den vorschulischen Bereich werden derzeit verschiedene Konzepte sprachlicher Bildung diskutiert. Allen gemein ist, dass es für ihre Umsetzung qualifizierte frühpädagogische Fachkräfte mit ausgeprägten professionellen Kompetenzen bedarf (Dickinson und Porche 2011; Jungmann et al. 2013). In den letzten Jahren wurden diese Kompetenzen im Rahmen von professionellen Kompetenzmodellen näher beschrieben (Mischo und Fröhlich-Gildhoff 2011; Anders 2012; Blossfeld 2012). Neben dem Fachwissen und dem fachdidaktischen Wissen haben sich für die Umsetzung von sprachlicher Bildung auch die Überzeugungen von Fachkräften sowie ihre motivationalen Orientierungen als bedeutsam erwiesen (Kratzmann et al. 2017). Obwohl Überzeugungen - als subjektiv bedeutsames Bezugssystem im (früh)pädagogischen Fachdiskurs inzwischen eine zentrale Rolle für die Qualität der Anregungen innerhalb einer Einrichtung zugesprochen werden, gibt es bislang jedoch kaum Forschungen, die die bereichsspezifischen Aspekte wie Überzeugungen im Umgang mit kultureller Diversität in den Fokus nehmen und ihren $\mathrm{Zu}$ sammenhang mit motivationalen Orientierungen der Fachkräfte untersuchen. Als eine zentrale motivationale Orientierung für die Gestaltung einer lernförderlichen Praxis haben sich in der Schulforschung die Selbstwirksamkeitserwartungen der Lehrkräfte herausgestellt (Jerusalem und Hopf 2002; Zee und Koomen 2016). Allerdings liegen auch zu diversitätsspezifischen Selbstwirksamkeitserwartungen nur wenige Forschungsbefunde vor, die sich wiederum auf Lehrkräfte in Schulen beziehen (Hachfeld et al. 2012).

Der vorliegende Beitrag setzt hier an und untersucht, inwieweit multikulturelle Überzeugungen und diversitätsspezifische Selbstwirksamkeitserwartungen von frühpädagogischen Fachkräften miteinander in Beziehung stehen und ob sie sich im zeitlichen Verlauf verändern oder stabil bleiben. Ferner wird die prädiktive Bedeutung der multikulturellen Überzeugungen und diversitätsspezifischen Selbstwirksamkeits-

\footnotetext{
1 In gängigen Definitionen weisen Personen einen Migrationshintergrund auf, wenn sie selbst oder deren Eltern bzw. Großeltern nach Deutschland immigriert sind. Bei diesem breiter gefassten Migrationskonzept spielt die aktuelle Staatsangehörigkeit keine Rolle (vgl. Autorengruppe Bildungsberichterstattung 2018). Da sich der Fokus der vorliegenden Studie auf das Thema „Sprache“ konzentriert und die Staatsbürgerschaft der Eltern und des Kindes keine erziehungswissenschaftliche oder praktisch-pädagogisch relevante Kategorie beschreibt (Gogolin 2016), liegt nach unserem Verständnis dann ein Migrationshintergrund vor, wenn in einer Familie überwiegend eine andere Sprache als Deutsch gesprochen wird, wohlwissend, dass mit dieser Definition eine Vereinfachung in Kauf genommen wird.
} 
erwartungen für die eingeschätzte Wichtigkeit von zwei verschiedenen Sprachförderansätzen geprüft.

\section{Sprachlich-kulturelle Diversität als zentrale Herausforderung für die sprachpädagogische Arbeit}

Die oben beschriebenen Herausforderungen schlagen sich in der Arbeit der frühpädagogischen Fachkräfte nieder: diese müssen sich in ihrer Arbeit sowohl mit unterschiedlichen sprachlichen Voraussetzungen der Kinder und ihrer Familien als auch mit diversen normativen Erziehungsvorstellungen ${ }^{2}$ auseinandersetzen (Morgan 2016). Durch eine wertschätzende Kommunikation können Fachkräfte jedoch als wichtige Vertrauenspersonen und als Bindeglied zwischen Familie und Kind fungieren. Insbesondere für zugewanderte Familien kann eine positive Beziehung zur Kindertageseinrichtung die Integration in die Gesellschaft erleichtern (Schlösser 2012), denn die Mehrheit der Kinder mit Migrationshintergrund, die vorschulische Bildungseinrichtungen besuchen, spricht zu Hause überwiegend eine andere Sprache als Deutsch (Autorengruppe Bildungsberichterstattung 2018). Für diese Kinder können Kindertageseinrichtungen entscheidende Wegbereiter der Sprachförderung im Deutschen sein. Studien weisen positive Effekte eines frühzeitigen Eintritts in Einrichtungen der frühkindlichen Bildung für Kinder mit Migrationshintergrund nach (Anders 2013; Roßbach et al. 2009).Viele Fachkräfte erleben die Zusammenarbeit mit Familien mit Migrationshintergrund jedoch oft als konflikthaft (Sulzer 2013), glauben, den Anforderungen aufgrund der zunehmenden kulturellen Diversität nicht gerecht zu werden (Neumann 2005) und wünschen sich vermehrt Fortbildungen zu dieser Thematik (Schweitzer et al. 2011).

Der Unsicherheit von Fachkräften steht bei vielen zugewanderten Familien (insbesondere der ersten Generation) fehlendes Wissen über das frühkindliche Bildungsund Erziehungssystem gegenüber. Damit sind unter anderem Informationen über die Vergabe von Betreuungsplätzen angesprochen, die neben den allgemeinen sozialstrukturellen Zugangs- und Partizipationsmöglichkeiten der Familien eine entscheidende Rolle bei der Wahl bzw. der Vergabe eines Kitaplatzes spielen (Sachverständigenrat Deutscher Stiftungen für Integration und Migration (SVR) 2013). So konnte bei der Wahl des Kitaplatzes beispielsweise für türkische Familien nachgewiesen werden, dass sie den in ihrem Wohngebiet vorgegebenen Möglichkeiten stärker folgen als deutsche Familien und ihre Kinder somit häufiger in Kindertageseinrichtungen mit einem höheren Anteil anderer Kinder mit Migrationshintergrund anmelden (Biedinger und Becker 2010). Aus dem Besuch hoch segregierter Kindertageseinrichtungen resultieren für die Kinder wiederum unterschiedliche Bedingungen für den Erwerb der deutschen Sprache und weiterer Kompetenzen (Becker 2006, 2010; Lehrl et al. 2014). Einige Befunde belegen, dass ein hoher Anteil von Kindern mit Migrationshintergrund in den Gruppen oftmals mit einer geringeren

\footnotetext{
2 Es kann davon ausgegangen werden, dass sich die normativen Vorstellungen von Familien mit Migrationshintergrund je nach Zuwanderergeneration deutlich unterscheiden (vgl. Sachverständigenrat Deutscher Stiftungen für Integration und Migration (SVR) 2013).
} 
pädagogischen Qualität einher geht (Kuger und Kluczniok 2009; Tietze et al. 2013) und sich negativ auf den Erwerb der deutschen Sprache auswirkt (Ebert et al. 2013; Tietze et al. 2013).

Gleichwohl legen Befunde aus der amerikanischen Schulforschung den Schluss nahe, dass ein Mehr an sprachlichem Input nicht zwangsläufig zu einem Fortschritt der sprachlichen Kompetenzen führt (McLaughlin und McLeod 1997). Vielmehr sollten interessengeleitete Anreize für Kinder geschaffen werden, die scheinbar naturwüchsig die sprachlichen Aktivitäten in Gang setzen (Jampert 2002; für einen Überblick, welche Faktoren für den Zweitsprachenerwerb eine Rolle spielen, siehe Piske 2014). Als Erfolg versprechende vorschulische Konzepte sprachlicher Bildung werden unter Forschenden und Praktizierenden in Deutschland derzeit die additive sprachpädagogische Förderung und die alltagsintegrierte sprachliche Bildung diskutiert.

\subsection{Additive sprachpädagogische Förderung}

Additive Sprachförderprogramme werden zusätzlich zu den alltäglichen Kita-Angeboten umgesetzt und dienen vornehmlich der Kompensation bereits früh bestehender sprachlicher Diskrepanzen. Additive Sprachförderprogramme adressieren Kinder mit spezifischem Sprachförderbedarf. Studien zeigen, dass Kinder mit nichtdeutscher Familiensprache und Kinder aus bildungsfernen Familien in solchen Programmen häufig überrepräsentiert sind (Welch-Sing und Pagonis 2011; Gagarina et al. 2014). Additive Sprachförderprogramme zeichnen sich weiterhin dadurch aus, dass sie außerhalb des regulären Gruppengeschehens stattfinden, einen hohen Strukturierungsgrad aufweisen sowie inhaltlich auf spezifische Teilkompetenzen des (Schrift-)Spracherwerbs fokussieren (Petermann 2015). Die Wirksamkeit diverser additiver Sprachförderprogramme auf die kindliche Sprachentwicklung konnte anhand des bisherigen Forschungsstandes nicht nachgewiesen werden, da sich nach systematischer Überprüfung entweder keine oder nur sehr geringe Effekte ermitteln ließen (Hofmann et al. 2008; Wolf et al. 2011; Sachse et al. 2012). Positive Effekte konnten für einen engen Teilbereich der Sprachentwicklung - der phonologischen Bewusstheit - aufgezeigt werden (Fischer und Pfost 2015), allerdings scheinen sich die frühen Vorteile in dieser Sprachkomponente nicht auf die Entwicklung weiterer sprachlicher Komponenten im Deutschen auszuwirken (Pfost 2015). Eine Problematik bei der Umsetzung additiver Sprachförderprogramme stellt die konzept-getreue Durchführung dar, die häufig nur durch externe Fachkräfte sichergestellt werden kann (Sachse 2001; Schröder 2012). Die bisherigen Ergebnisse zu additiven Sprachförderprogrammen lassen keine eindeutigen Schlussfolgerungen zu, zumal sie häufig nur mit unzureichender Implementationsqualität umgesetzt werden (Kammermeyer und Roux 2013) und schwierige strukturelle Rahmenbedingungen eine erfolgreiche Umsetzung beeinträchtigen können (Viernickel et al. 2013). Die bislang nicht nachgewiesene Wirksamkeit könnte auch damit zusammenhängen, dass die Programmdauer zu kurz oder die Intensität zu gering ausfiel und nicht an der Qualität der Programme oder der additiven Förderausrichtung. 


\subsection{Altagsintegrierte sprachliche Bildung}

Das Konzept der alltagsintegrierten sprachlichen Bildung umfasst verschiedene Sprachförderstrategien, wie z.B. das dialogische Bilderbuchlesen oder das korrektive Feedback. Alltagsintegrierte Sprachförderstrategien sind systematisch in das alltägliche Geschehen der Kindertageseinrichtungen eingebettet und orientieren sich situationsorientiert an den Bedürfnissen und Interessen aller Kinder. Das Konzept erweitert den Blick auf Sprache und spricht die Sprachentwicklung in ihrer Ganzheitlichkeit an. So sind Fachkräfte angeleitet, Alltagssituationen in der Form zu gestalten, dass sie geeignete sprachliche Anregungen für den beiläufigen und spielerischen Kompetenzerwerb der Kinder bereitstellen (Hasselhorn und Sallat 2014). Um diese Funktion des sprachlichen Vorbildes auszufüllen, sind besondere Anstrengungen und fundiertes Fachwissen über Spracherwerbs- bzw. Entwicklungsprozesse nötig. In ihrer Übersichtsarbeit stufen Egert und Hopf (2016) alltagsintegrierte Sprachförderkonzepte insbesondere für Kinder unter drei Jahren vorläufig als wirkungsvoll ein, während die Befunde zu additiven Sprachförderkonzepten inkonsistent sind. So belegt die Studie von Buschmann und Joos (2011) stärkere Zuwächse im Wortschatz und der sprachproduktiven Leistungen der zweijährigen Kinder aus der Interventionsgruppe verglichen mit den Kindern aus der Kontrollgruppe. Auch Anders et al. (2016) konnten Effekte der sprachbezogenen pädagogischen Qualität der Kindertageseinrichtungen auf die sprachliche Entwicklung der Kinder im Deutschen nachweisen. Ferner konnten Simon und Sachse (2013) Fördereffekte in den Kommunikationsanteilen bei den Kindern und eine gesteigerte Komplexität der kindlichen Äußerungen infolge des Trainings des sprachlichen Interaktionsverhaltens der frühpädagogischen Fachkräfte feststellen. Als besonders förderliches Element für die Sprachentwicklung erwies sich im Vorschulalter die „Bilderbuch-Methode“ (Jungmann et al. 2013).

Trotz einiger erfolgsversprechender Befunde lassen auch die Studien zu alltagsintegrierten Sprachförderkonzepten bis dato keine allgemein gültigen Schlüsse über die Wirksamkeit zu. Grundsätzlich lassen sich Effekte alltagsintegrierter Sprachförderung auf die kindliche Sprachentwicklung aufgrund der Vielzahl an unterschiedlichen Umsetzungsvarianten nur schwer erfassen. Es bleibt festzuhalten, dass sowohl das Konzept der alltagsintegrierten als auch das der additiven Sprachförderung anspruchsvoll sind und deren Ausgestaltung und praktische Umsetzung von den frühpädagogischen Fachkräften umfangreiche Kompetenzen verlangen.

\section{Professionelle Kompetenzen als Handlungsvoraussetzung für pädagogische Prozessqualität}

In pädagogischen Handlungsfeldern ist von professionellen Kompetenzen die Rede, um Kenntnisse, Denkmuster, Fähigkeiten und Fertigkeiten zur Problemlösung komplexer Handlungssituationen im beruflichen Kontext zu beschreiben. Im frühpädagogischen Fachdiskurs wurden in den vergangenen Jahren auf Basis der gestiegenen Anforderungen an die Fachkräfte ebenfalls professionelle Kompetenzmodelle abgeleitet (Fröhlich-Gildhoff et al. 2011; Anders 2012). Als zentrale Kompetenzen 
werden in diesen mehrdimensionalen Modellen Professionswissen, pädagogische Orientierungen und Überzeugungen, motivationale und emotionale Aspekte sowie Aspekte des Selbst- und Rollenverständnisses genannt. Aufgrund ihrer handlungsleitenden Implikationen können Überzeugungen einen entscheidenden Beitrag für die sprachpädagogische Arbeit auf Einrichtungsebene leisten.

Überzeugungen als Bestandteil der professionellen Haltung frühpädagogischer Fachkräfte wirken zum einen als ein subjektiv bedeutsames Bezugssystem für das individuelle Denken und Handeln und zum anderen können sie als Wahrnehmungsfilter aufgefasst werden, da sie auf die Wahrnehmung und Verarbeitung neuer Informationen einwirken (Fives und Buehl 2012; Wischmeier 2012; Schroeter 2014). Im Gegensatz zum Wissen spiegeln Überzeugungen Bewertungen, Meinungen, Eindrücke oder subjektive Erklärungssysteme wider (Kunter und Trautwein 2013). Sie implizieren einerseits einen hohen Anteil an biografischen Erfahrungen, wodurch sie einen dezidiert, subjektiv bedeutsamen Wahrheitsgehalt für die Person besitzen. Andererseits werden Überzeugungen auch durch soziales Wissen, das über die Kommunikation mit anderen Personen gewonnen wird, beeinflusst (Abelson 1979; Nespor 1987). Pädagogische Überzeugungen im Speziellen stellen eine wichtige Entscheidungsgrundlage für das eigene professionelle Handeln dar und implizieren zudem einen Wertebezug (Tietze und Meischner 1998). Sie repräsentieren ein breites Konstrukt, das unterschiedliche Teilaspekte wie beispielsweise Überzeugungen zu frühpädagogischen Ansätzen oder auch zum Bildungsbereich Sprache in Kindertageseinrichtungen umfassen (Anders 2012). Da pädagogische Überzeugungen als Rahmenbedingungen für das direkte pädagogische Handeln fungieren, wird ihnen ein Einfluss auf die Interaktionen zwischen Fachkraft und Kind bzw. Familie unterstellt (Tietze und Meischner 1998; Pianta et al. 2005). Damit sich Überzeugungen in Handlungen niederschlagen können, sind allerdings noch weitere Kompetenzfacetten sowie gegebenenfalls strukturelle Voraussetzungen notwendig. Schließlich spielen eine Vielzahl unterschiedlicher Faktoren, wie beispielsweise der situative Kontext, die Zielsetzung, die Befähigung oder auch die Motivation, für die Umsetzung eines überzeugungsgerechten Handelns eine Rolle. Bislang gibt es nur wenige empirische Untersuchungen, die einen Einfluss von pädagogischen Orientierungen vermittelt über die Prozessqualität - auf die kindliche Entwicklung aufzeigen konnten (Kluczniok et al. 2011). In Bezug auf die sprachpädagogische Förderung liegen einerseits Befunde vor, nach denen positive Überzeugungen gegenüber Mehrsprachigkeit in Kindertagesstätten für eine alltagsintegrierte Sprachförderung förderlich sind (Kratzmann et al. 2017). Andererseits konnte auch aufgezeigt werden, dass positive Überzeugungen zum Einbezug der Erstsprache auf Seiten der Fachkräfte mit einer schlechteren deutschsprachigen Entwicklung von Kindern mit spezifischem Sprachförderbedarf assoziiert sind (Kratzmann et al. 2013).

Pädagogische Überzeugungen sind eng mit Selbstwirksamkeitserwartungen verknüpft, die zu den motivationalen und emotionalen Aspekten des professionellen Handelns gezählt werden können (Anders 2012). Selbstwirksamkeitserwartungen lassen sich definieren als das Vertrauen einer Person, neue und komplexe Anforderungen auf Grundlage der eigenen Kompetenzen bewältigen zu können (Bandura 1997, 2001). Personen ohne Vertrauen in die eigenen Kompetenzen würden entsprechend herausfordernde Situationen vermeiden und stärker zu sozial unsicherem 
Verhalten sowie Routinehandlungen tendieren. Je nach Lebensbereich und Situation können unterschiedliche Grade an Selbstwirksamkeitserwartungen ausgebildet sein. Aufgrund dessen werden bereichsspezifische Aspekte der Selbstwirksamkeitserwartungen, wie beispielsweise die Kompetenz, sprachliche Bildungsprozesse in Gruppen von Kindern unterschiedlicher kultureller Herkunft angemessen zu begleiten, voneinander unterschieden.

\subsection{Multikulturelle Überzeugungen}

Multikulturelle Überzeugungen ${ }^{3}$ - als Bestandteil der Überzeugungen einer Person über kulturelle Diversität - bezeichnen eine wertschätzende Haltung gegenüber unterschiedlichen Kulturen (Hachfeld et al. 2012). Pädagogische Fachkräfte mit ausgeprägten multikulturellen Überzeugungen heben eine interkulturelle Dimension in Bildungsprozessen hervor und sie berücksichtigen kulturelle und sprachliche Diversität, wenn sie in Gruppen oder mit einzelnen Kindern interagieren. In der pädagogischen Praxis zeichnet sich diese Haltung der Fachkräfte durch das Eingehen auf unterschiedliche kulturelle Wertesysteme aus. Dabei wird das eigene Wertsystem nicht negiert und auch Spannungen in der pädagogischen Praxis bleiben nicht aus. Gleichwohl sind sich Fachkräfte mit ausgeprägten multikulturellen Überzeugungen der kulturellen Unterschiede und des daraus resultierenden möglichen Konfliktpotenzials bewusst. Sie negieren diese nicht, sondern machen sie als Ressource in der Interaktion mit den Kindern pädagogisch nutzbar. Stärker ausgeprägte multikulturelle Überzeugungen schärfen somit die Sensibilität gegenüber den eigenen Vorurteilen und vorschnellen Verallgemeinerungen. Dies setzt bei den Fachkräften einen (selbst-)kritischen Reflexionsprozess voraus. Nur dadurch lassen sich Hinweise auf die eigene kulturelle Gebundenheit und das pädagogisch angemessene Verhalten erkennen.

Forschungsergebnisse deuten darauf hin, dass Personen mit stark ausgeprägten multikulturellen Überzeugungen sensibler und vorurteilsbewusster mit kulturellen Unterschieden umgehen als Personen, die verstärkt die Gemeinsamkeiten und weniger den kulturellen Hintergrund fokussieren (Wolsko et al. 2006; Vorauer et al. 2009). So berichteten Lehramtsanwärterinnen in einer Studie von Hachfeld et al. (2015) eine umso höhere Bereitschaft, in ihrem Unterricht auf kulturelle Unterschiede einzugehen, je mehr sie multikulturellen Überzeugungen zustimmten. Anders et al. (2016) konnten darüber hinaus nachweisen, dass die multikulturellen Überzeugungen der zusätzlichen Fachkräfte für sprachliche Bildung einen Einfluss auf die sprachbezogene Prozessqualität in den Kindertageseinrichtungen hatten. Auch für die Identitätsentwicklung von Kindern spielt es eine wichtige Rolle, wie die pädagogischen Fachkräfte mit kultureller Diversität umgehen und ob sich Kinder als

\footnotetext{
3 Es existiert in der deutschsprachigen Forschung bislang noch keine allgemein gültige Definition, um Überzeugungen zu kultureller Diversität zu spezifizieren. So gibt es große Überschneidungsbereiche zu verwandten Konzepten, wie beispielsweise zu dem Einstellungsbegriff (vgl. van Dick et al. 1997; Kratzmann et al. 2017). Im US-amerikanischen Forschungsbereich zu kultureller Diversität hat sich der Begriff der „Überzeugungen“ (beliefs) etabliert (vgl. Bryan und Atwater 2002; Gay 2010), auf den wir uns im Weiteren beziehen werden. Wir verwenden damit den Begriff „Überzeugungen“ äquivalent zu dem der „Einstellungen“.
} 
normal wahrgenommen fühlen. Kinder, die aus Familien stammen, die den Merkmalen der Mehrheitsgesellschaft nicht entsprechen, beschäftigen sich häufiger mit Fragen ethnischer Zuordnung und ihrem eigenen Bild als Kinder, deren Familien den Merkmalen der Mehrheitsgesellschaft entsprechen (Lane 2008; MacNaughton und Davis 2009). Im Vergleich entwickeln letztere eher Gefühle von Überlegenheit (Derman-Sparks und Ramsey 2006; MacNaughton und Davis 2009). Die multikulturellen Überzeugungen der pädagogischen Fachkräfte können daher einen bedeutenden Beitrag für ein differenziertes, vorurteilsfreies und wertschätzendes Verständnis ethnisch-kultureller Diversität in Kindertageseinrichtungen leisten.

\subsection{Diversitätsspezifische Selbstwirksamkeitserwartungen}

Selbstwirksamkeitserwartungen stellen eine zentrale Voraussetzung für kompetente, selbstregulative Handlungsprozesse dar. In der pädagogisch-psychologischen Forschung wurde ihre Bedeutung für die Erklärung von Lern- und Leistungsprozessen vielfach nachgewiesen (Jerusalem und Hopf 2002; Parker et al. 2006). Für den pädagogischen Kontext werden Lehrende mit ausgeprägten Selbstwirksamkeitserwartungen gerade für die Förderung von lernschwachen Schülerinnen und Schülern mit niedrigem Sprachniveau häufig als Desiderat gefordert, da sie den Bedürfnissen dieser Gruppe eher gerecht werden können (Schwarzer und Jerusalem 2002; Ross und Bruce 2007). So setzen sie sich zum einen selbst höhere Ziele und sind zum anderen auch stärker motiviert, diese Schülerinnen und Schüler bei der Erreichung ihrer Lernziele zu unterstützen. Das Vertrauen in die Fähigkeiten aller Kinder unabhängig von deren sozialem und kulturellem Hintergrund - ist insbesondere für den Bildungsverlauf von Kindern mit Migrationshintergrund wichtig, da sie häufig ohnehin spezifischen Risikolagen ausgesetzt sind. Für den Vorschulbereich konnten positive Interaktionseffekte zwischen den Selbstwirksamkeitserwartungen der Lehrkräfte und der emotionalen Unterstützung sowie der Wortschatzerweiterung bei den betreuten Kindern aufgezeigt werden (Guo et al. 2010). Ferner erwiesen sich Selbstwirksamkeitserwartungen in Bezug auf Unterrichtsführung und Schülermotivation als ein bedeutender Prädiktor für die Qualität der Literacy-Förderung (Justice et al. 2008).

Selbstwirksamkeitserwartungen sind immer an einen spezifischen Kontext gebunden und lassen sich entsprechend nicht verallgemeinern (Bandura 1997; TschannenMoran und Hoy 2001). So können pädagogische Fachkräfte grundsätzlich über allgemein ausgeprägte Selbstwirksamkeitserwartungen verfügen, sich aber gleichzeitig unsicher im Umgang mit Kindern mit Migrationshintergrund fühlen. Personen mit ausgeprägten diversitätsspezifischen Selbstwirksamkeitserwartungen trauen sich $\mathrm{zu}$, Kinder mit Migrationshintergrund in ihren spezifischen Bedürfnissen zu fördern (z.B. in der Verbesserung ihrer sprachlichen Fähigkeiten im Deutschen) und sie spornen sie zum Erreichen der kommenden Entwicklungsschritte an. Bislang gibt es allerdings nur wenige Forschungsbefunde zu Selbstwirksamkeitserwartungen im Umgang mit kultureller Diversität. Hachfeld et al. (2012) konnten bei Lehramtsstudierenden einen positiven Zusammenhang zwischen deren multikulturellen Überzeugungen und den Selbstwirksamkeitserwartungen für das Unterrichten von Schülerinnen und Schüler mit Migrationshintergrund aufzeigen. Ferner konnte bei 
Lehramtsstudierenden ein negativer Zusammenhang zwischen der Selbstwirksamkeit bezüglich des kultursensiblen Classroom Managements und dem Stressempfinden durch kulturelle Diversität nachgewiesen werden (Civitillo et al. 2016a). Amerikanische Forschungsbefunde legen nahe, dass Lehrkräfte, die sich in der Lage fühlen, kulturell ansprechende Lehraufgaben erfolgreich durchzuführen, auch entsprechende pädagogische Praktiken in der Praxis anwenden (Siwatu 2007). Fehlt dieses Vertrauen in die eigene Fähigkeit, hilft es auch nicht, wenn der Wert und der Nutzen kulturell ansprechender Unterrichtspraktiken erkannt wird (Siwatu et al. 2016).

\subsection{Stabilität und Veränderbarkeit von Überzeugungen und Selbstwirksamkeitserwartungen}

Im Rahmen der Debatte über professionelle Kompetenzen frühpädagogischer Fachkräfte wird von der Erlern- bzw. Veränderbarkeit von Überzeugungen und motivationalen Orientierungen durch Aus-, Fort- sowie Weiterbildungsmöglichkeiten ausgegangen (Tietze und Meischner 1998; Anders 2012). Um Veränderungen herbeizuführen, bedarf es allerdings spezifischer Voraussetzungen, da Überzeugungen und Selbstwirksamkeitserwartungen grundsätzlich als zeitlich stabil angesehen werden. Entsprechend können einmal in der Jugend verinnerlichte Attributionsmuster noch im hohen Erwachsenenalter beibehalten werden (Burns und Seligman 1989).

Überzeugungen implizieren einerseits einen großen Anteil an biografischen Erfahrungen, wodurch ihnen ein hoher, subjektiv bedeutender Wahrheitsgehalt eigen ist; andererseits werden Überzeugungen aber auch durch soziales Wissen beeinflusst, das über die Kommunikation mit anderen Personen gewonnen wird (Abelson 1979; Nespor 1987). Gemäß der Kontakthypothese (Pettigrew 1998) führen interethnische Kontakte unter bestimmten Voraussetzungen zu positiven Effekten auf Überzeugungen gegenüber Migrantengruppen. Als eine wichtige Voraussetzung erweist sich dabei der spezifische Migrantenanteil: Wird ein bestimmter Schwellenwert überschritten, so nehmen Vorurteile wieder zu (Wagner et al. 2006; Weins 2011). Civitillo et al. (2016b) konnten in einem Übersichtsartikel aufzeigen, dass positive Veränderungen in den multikulturellen Überzeugungen von angehenden Lehrkräften durch Trainingsmodule insbesondere dann eintrafen, wenn neben theoretischen Inhalten auch der direkte Austausch mit Menschen unterschiedlicher kultureller Herkunft gegeben war. Als dritte entscheidende Komponente zur Effektivitätssteigerung der Module wird in dem Artikel die Möglichkeit zur Selbstreflexion identifiziert. Für den deutschen Kontext konnte für Lehrkräfte die Bedeutung interkultureller Lebenserfahrung für die Entstehung von multikulturellen Überzeugungen (Göbel und Helmke 2010) und die inhaltliche Qualität interkulturellen Unterrichts aufgezeigt werden (Göbel und Hesse 2008).

Die Bedeutung des interethnischen Kontaktes konnte auch für die Veränderung von diversitätsspezifischen Selbstwirksamkeitserwartungen nachgewiesen werden. So konnte Bender-Szymanski (2000) in einer qualitativen Längsschnittstudie aufzeigen, dass angehende Lehrkräfte, die ihr eigenes Verhalten bei kulturellen Differenzen mit Schülerinnen und Schüler unterschiedlicher kultureller Herkunft reflektieren und die Konflikte nicht als persönlichen Angriff auffassen, ihre Selbstwirksamkeits- 
erwartungen nach einem Jahr höher einschätzen als zu Studienbeginn. Demgegenüber berichtet diejenige Gruppe angehender Lehrkräfte, die die Werte ihrer eigenen Kultur als unbestrittenes und einziges Beurteilungskriterium bei Differenzen heranziehen, über abnehmende Selbstwirksamkeitserwartungen. Die Veränderbarkeit von Selbstwirksamkeitserwartungen konnte auch in anderen Studien nachgewiesen werden (Kopp 2009). Sie können somit grundsätzlich gefördert werden, wobei als einflussreichste Quellen Erfahrungen von Erfolg eigener Handlungen, stellvertretende Erfahrungen durch Beobachtung, verbale Überzeugungen sowie Wahrnehmungen eigener physiologischer Erregung gelten (Bandura 1997, 2001).

\section{Forschungsanliegen}

In dieser Studie wird der Frage nachgegangen, inwieweit die multikulturellen Überzeugungen mit den diversitätsspezifischen Selbstwirksamkeitserwartungen der frühpädagogischen Fachkräfte - auch über den zeitlichen Verlauf - in Zusammenhang stehen (Forschungsfrage 1a). Wir gehen auf Basis von Handlungskompetenzmodellen (Fröhlich-Gildhoff et al. 2011; Anders 2012) davon aus, dass die beiden Kompetenzfacetten eng miteinander in Beziehung stehen, sie aber gleichzeitig zwei trennbare Konstrukte darstellen. Neben der wechselseitigen Beziehung wird auch untersucht, ob die multikulturellen Überzeugungen und diversitätsspezifischen Selbstwirksamkeitserwartungen sich über den zeitlichen Verlauf verändern oder sich eher durch eine zeitliche Invarianz auszeichnen (Fragestellung 1b). Da es sich bei der von uns untersuchten Stichprobe um frühpädagogische Fachkräfte handelt, die während des Untersuchungszeitraums regelmäßig an Fortbildungen zu handlungs- bzw. situationsorientierter sprachlicher Bildung für Kinder mit speziellen Förderbedarfen teilgenommen haben (siehe 5.1), erwarten wir über den zeitlichen Verlauf tendenzielle Veränderungen.

Im Rahmen der zweiten Forschungsfrage wird untersucht, ob die multikulturellen Überzeugungen und die diversitätsspezifischen Selbstwirksamkeitserwartungen der frühpädagogischen Fachkräfte mit der von ihnen eingeschätzten Wichtigkeit zweier sprachpädagogischer Förderstrategien längsschnittlich in Zusammenhang stehen. Sind die beiden Kompetenzfacetten zum ersten Messzeitpunkt mit einer stärkeren Zustimmung zu alltagsintegrierten oder additiven sprachpädagogischen Förderstrategien zum zweiten Messzeitpunkt assoziiert (Fragestellung 2a und 2b)? Alltagsintegrierte und additive sprachpädagogische Förderstrategien bilden unter Forschenden und Praktizierenden zwei relevante und umstrittene Konzepte ab, zumal die bisherigen Befunde keine allgemein gültigen Schlussfolgerungen über das Primat eines der beiden Konzepte zulassen. Arbeiten zur Struktur der Überzeugungen sprechen für unterscheidbare, aber miteinander korrelierte Überzeugungsfacetten (Wieduwilt et al. submitted). Eine Dichotomisierung erscheint dementsprechend artifiziell. Auch aus einer präventionstheoretischen Perspektive sind beide Konzepte notwendig und sollten sich ergänzen (Hasselhorn und Sallat 2014). Kompetenzen, die die Umsetzung beider Strategien fördern, sollten sich daher positiv auf die Sprachentwicklung der Kinder auswirken. Wir gehen davon aus, dass sowohl ausgeprägte multikulturelle Überzeugungen als auch diversitätsspezifische Selbstwirksamkeitserwartungen 
von kultursensitiven pädagogischen Fachkräften in Zusammenhang mit der eingeschätzten Wichtigkeit alltagsintegrierter und additiver sprachpädagogischer Förderstrategien stehen.

\section{Methode}

Grundlage der Untersuchung sind Längsschnittdaten, die im Rahmen der Evaluation des Bundesprogramms „Schwerpunkt-Kitas Sprache \& Integration“ in dem Untersuchungszeitraum von Januar 2012 bis Dezember 2015 erhoben wurden (Roßbach et al. 2016). „Schwerpunkt-Kitas Sprache \& Integration“ war ein vom Bundesministerium für Familie, Senioren, Frauen und Jugend (BMFSFJ) initiiertes Programm für den frühkindlichen Bereich. Der Fokus des Programms lag primär auf Kindertageseinrichtungen, die sich durch einen überdurchschnittlich hohen Anteil von benachteiligten Kindern oder Kindern mit Migrationshintergrund sowie Kindern unter drei Jahren auszeichneten. Im Rahmen des Bundesprogramms erhielten 4000 teilnehmende Einrichtungen eine zusätzliche Fachkraft für sprachliche Bildung (im Folgenden: Sprachexpertin ${ }^{4}$ ) mit Halbtagsbeschäftigung sowie Zuwendungen ${ }^{5}$ in Form von Sachkosten (z. B. Fortbildungen, Lehr- bzw. Lernmaterialien) und Gemeinkosten (z.B. anteilige Mietkosten). Diese Sprachexpertinnen sollten als Multiplikatorinnen in das Team hineinwirken und dieses weiterentwickeln, um allen Kindern alltagsintegrierte sprachliche Bildung zugänglich zu machen und so zu einer langfristigen Qualitätsverbesserung der Einrichtungen beizutragen. Die Evaluation untersuchte die Implementation und die Auswirkungen des Bundesprogramms auf den Ebenen der Einrichtungen, Fachkräfte und Teams sowie der Familien und Kinder (Roßbach et al. 2016). In dieser Studie stehen nicht die evaluativen Fragestellungen im Zentrum, sondern die oben beschriebenen.

\subsection{Stichprobe}

In die Analyse gingen Daten von denjenigen Sprachexpertinnen $(N=251)$ ein, die zu zwei Messzeitpunkten (Winter 2012; Herbst 2014) online befragt wurden und über diesen Zeitraum in derselben Einrichtung beschäftigt waren. Die Sprachexpertinnen wiesen vertiefte Erfahrungen in der sprachlichen Bildungsarbeit auf und nahmen im Untersuchungszeitraum von Januar 2012 bis Dezember 2015 regelmäßig an Fortbildungen zum Thema alltagsintegrierte sprachliche Bildung teil. Es handelt sich somit um eine spezielle Stichprobe von sprachpädagogisch vergleichsweise hoch qualifizierten Fachkräften.

\footnotetext{
4 Aus Gründen der besseren Lesbarkeit wird nicht explizit nach geschlechtsspezifischen Personenbezeichnungen differenziert. Wir wählten die weibliche Form der Bezeichnungen, da die überwiegende Mehrheit der untersuchten zusätzlichen Fachkräfte Frauen sind $(n=244 ; 97,2 \%)$. Die gewählte weibliche Form schließt die männlichen Fachkräfte gleichberechtigt ein.

5 Für einen genauen Überblick über die Auswahlkriterien und Fördermaßnahmen siehe Bundesministerium für Familie, Senioren, Frauen und Jugend (BMFSFJ) (2015).
} 
Tab. 1 Biographische und berufsspezifische Merkmale der Sprachexpertinnen sowie Anteil an Kindern mit Migrationshintergrund in der Einrichtung

\begin{tabular}{|c|c|c|c|c|c|c|}
\hline & $N$ & $M$ & $S D$ & Min & $\operatorname{Max}$ & $\begin{array}{l}\text { Anteil } \\
(\text { Anzahl })\end{array}$ \\
\hline \multicolumn{7}{|l|}{ Fachkraftebene } \\
\hline Alter & 251 & 41,38 & 9,39 & 22 & 65 & - \\
\hline Berufserfahrung (Jahre) & 251 & 11,65 & 9,98 & 0 & 42 & - \\
\hline Erstsprache nicht deutsch & 247 & - & - & - & - & $9,31 \%(23)$ \\
\hline Mit Abitur & 251 & - & - & - & - & $23,90 \%(60)$ \\
\hline Mit (Fach-)Hochschulabschluss & 251 & - & - & - & - & $26,69 \%(67)$ \\
\hline \multicolumn{7}{|l|}{ Einrichtungsebene } \\
\hline $\begin{array}{l}\text { Anteil Kinder mit Migrationshin- } \\
\text { tergrund }\end{array}$ & 188 & 43,80 & 26,21 & 0 & 100 & - \\
\hline
\end{tabular}

In Tab. 1 sind die deskriptiven Statistiken aufgeführt. Der Migrationshintergrund der Sprachexpertinnen und Kinder wurde anhand der Erstsprache operationalisiert. Die Berufserfahrung bezieht sich auf Einrichtungen der frühkindlichen Bildung, Erziehung, und Betreuung.

\subsection{Instrumente}

Die eingesetzten Skalen wurden im Rahmen der Evaluation des Bundesprogramms „Schwerpunkt-Kitas Sprache \& Integration“ entwickelt (Anders et al. 2016) bzw. für die Studie angepasst (Hachfeld et al. 2011, 2012).

Multikulturelle Überzeugungen: Die Skala der multikulturellen Überzeugungen umfasste fünf Items, die einen kultursensitiven Umgang mit Kindern und Familien mit Migrationshintergrund erfassen (Beispielitem: „Im Rahmen der Arbeit mit Kindern mit Migrationshintergrund ist es wichtig, auch auf Unterschiede von verschiedenen Kulturen einzugehen "). Die Items konnten auf einer sechsstufigen Antwortskala von gar nicht zutreffend (1) bis voll zutreffend (6) beantwortet werden. Die interne Konsistenz der Skala lag zum ersten Messzeitpunkt bei $\alpha=0,77$ und zum zweiten Messzeitpunkt bei $\alpha=0,87$. Die Faktorladungen reichten zum ersten Messzeitpunkt von 0,52 bis 0,76 und zum zweiten Messzeitpunkt von 0,66 bis 0,83.

Diversitätsspezifische Selbstwirksamkeitserwartungen: Die Skala der diversitätsspezifischen Selbstwirksamkeitserwartungen beinhaltete vier Items, die auf das subjektive Vertrauen abzielen, Anforderungssituationen mit Kindern mit Migrationshintergrund erfolgreich bewältigen zu können (Beispielitem: „Ich bin zuversichtlich, dass ich auf die verschiedenen Belange von Kindern mit Migrationshintergrund eingehen kann"). Die Items konnten auf einer sechsstufigen Antwortskala von gar nicht zutreffend (1) bis voll zutreffend (6) beantwortet werden. Die interne Konsistenz der Skala lag zum ersten Messzeitpunkt bei $\alpha=0,85$ und zum zweiten Messzeitpunkt bei $\alpha=0,90$. Die Faktorladungen reichten zum ersten Messzeitpunkt von 0,70 bis 0,82 und zum zweiten Messzeitpunkt von 0,79 bis 0,87 . 
Sprachpädagogische Förderstrategien: Die Subskala zu den alltagsintegrierten sprachpädagogischen Förderstrategien umfasste 6 Items (Beispielitem: ,, Sprachpädagogische Arbeit mit Kindern sollte über das Nacherzählen von Geschichten stattfinden") und die Subskala zu den additiven sprachpädagogischen Förderstrategien 4 Items (Beispielitem: ,, Sprachpädagogische Arbeit mit Kindern sollte durch spezielle Sprachspiele stattfinden "). Die Teilnehmenden wurden gebeten, Sprachsituationen einzuschätzen, die sowohl alltagsintegrierte als auch additive sprachpädagogische Förderstrategien abbildeten. Alle Items konnten auf einer vierstufigen Antwortskala von unwichtig (1) bis wichtig (4) beantwortet werden. Die interne Konsistenz der Subskala „Wichtigkeit alltagsintegrierte sprachpädagogische Förderstrategien“ lag zum ersten Messzeitpunkt bei $\alpha=0,63$ und zum zweiten Messzeitpunkt bei $\alpha=0,62$. Die Faktorladungen reichten zum zweiten Messzeitpunkt von 0,33 bis 0,58. Die interne Konsistenz der Subskala ,Wichtigkeit additiver sprachpädagogischer Förderstrategien“" betrug zum ersten Messzeitpunkt $\alpha=0,77$ und zum zweiten Messzeitpunkt $\alpha=0,81$. Die Faktorladungen reichten zum zweiten Messzeitpunkt von 0,69 bis 0,74. Die Skalen korrelieren mit 0,66 $(p<0,01)$ miteinander.

\subsection{Analysen}

Die Fragestellungen wurden mithilfe von Strukturgleichungsmodellen in Mplus 7.4 (Muthén und Muthén 2015) untersucht. Es wurde final ein cross-lagged, autoregressives Modell spezifiziert. Dabei wurden die multikulturellen Überzeugungen, die diversitätsspezifischen Selbstwirksamkeitserwartungen und die Wichtigkeit alltagsintegrierter sprachpädagogischer Förderstrategien sowie die Wichtigkeit additiver sprachpädagogischer Förderstrategien latent modelliert. Zur Einschätzung der Modellgüte wurden vier Indizes herangezogen (Comparative Fit Index [CFI]; TuckerLewis-Index [TLI]; Root Mean Square Error of Approximation [RMSEA] sowie Standardized Root Mean Square Residual [SRMR]) und anhand der Kriterien von Hu und Bentler (1999) bewertet. Des Weiteren wurden der Chi-Quadrat-Wert und die dazugehörigen Freiheitsgrade $(d f)$ berücksichtigt. Fehlende Werte wurden über das Full-Information-Maximum-Likelihood-(FIML)-Verfahren geschätzt. Für den Anteil der Kinder mit Migrationshintergrund auf Einrichtungsebene lag er bei $25,1 \%$, bei allen anderen Variablen lediglich bei 1,6\% oder weniger.

In einem ersten Schritt wurde der Zusammenhang zwischen den multikulturellen Überzeugungen und den diversitätsspezifischen Selbstwirksamkeitserwartungen der Sprachexpertinnen untersucht (Forschungsfrage 1a). Neben den cross-lagged Beziehungen wurden simultan auch die autoregressiven Beziehungen der beiden Faktoren spezifiziert (Fragestellung 1b).

In einem zweiten Schritt wurde der Zusammenhang zwischen den multikulturellen Überzeugungen sowie den diversitätsspezifischen Selbstwirksamkeitserwartungen zum ersten Messzeitpunkt und der berichteten Wichtigkeit von alltagsintegrierten bzw. additiven sprachpädagogischen Förderstrategien zum zweiten Messzeitpunkt untersucht (Fragestellung 2a und 2b). Als Kontrollvariablen flossen die Berufserfahrung im frühpädagogischen Kontext (in Jahren), der Migrationshintergrund der Sprachexpertinnen sowie die Ausbildung ((Fach-)Hochschulstudium vorhanden/ 
nicht vorhanden) in die Analysen ein. Auf Einrichtungsebene wurde zusätzlich für den prozentualen Anteil an Kindern mit Migrationshintergrund kontrolliert.

\section{Ergebnisse}

\subsection{Deskriptive Ergebnisse}

Tab. 2 zeigt die deskriptiven und korrelativen Ergebnisse für die Variablen. Im Durchschnitt stimmten die Sprachexpertinnen den Aussagen zu den multikulturellen Überzeugungen sehr oder vollständig zu (MÜ MZP1: Spannweite von 3,40 bis 6,00; MÜ MZP2: Spannweite von 2,40 bis 6,00). Keine der befragten Fachkräfte nahm eine völlig ablehnende Haltung gegenüber einem kultursensitiven Umgang mit Kindern und Familien mit Migrationshintergrund ein. Sie wiesen zu beiden Messzeitpunkten auch hohe diversitätsspezifische Selbstwirksamkeitserwartungen auf (SE MZP1: Spannweite von 2,00 bis 6,00; SE MZP2: Spannweite von 2,50 bis $6,00)$.

Die deskriptiven Ergebnisse zu den Skalen zur Wichtigkeit alltagsintegrierter und additiver sprachpädagogischer Förderstrategien zeigten ein etwas differenzierteres Bild im Vergleich zu den multikulturellen Überzeugungen und den diversitätsspezifischen Selbstwirksamkeitserwartungen. Die Sprachexpertinnen maßen der alltagsintegrierten sprachpädagogischen Förderung im Durchschnitt zu beiden Messzeitpunkten eine hohe Bedeutung bei (AlS MZP1: Spannweite von 2,17 bis 4,00; AlS MZP2: Spannweite von 2,17 bis 4,00). Demgegenüber erachten sie die additiven sprachpädagogischen Förderstrategien als weniger wichtig (AdS MZP1: Spannweite von 1,25 bis 4,00; AdS MZP2: Spannweite von 1,25 bis 4,00). Dieser Unterschied fiel zu beiden Messzeitpunkten signifikant aus (MZP 1: $t=-11,86, d f=250$, MZP 2: $t=-13,94, d f=248)$.

\subsection{Der Zusammenhang zwischen multikulturellen Überzeugungen und diversitätsspezifischen Selbstwirksamkeitserwartungen}

In Abb. 1 ist das Strukturgleichungsmodell mit den yx-standardisierten Ergebnissen zum Zusammenhang zwischen multikulturellen Überzeugungen, diversitätsspezifischen Selbstwirksamkeitserwartungen sowie der Wichtigkeit sprachpädagogischer Förderstrategien aufgeführt. Unter den getesteten Modellen weist das aufgeführte Modell die beste Passung zu den Daten auf, was anhand der Modelgütekriterien und der Varianzaufklärung ersichtlich wird (vgl. Anmerkungen Abb. 1). Der Varianzaufklärungsanteil des Modells beträgt für die multikulturellen Überzeugungen 32,2\% (MZP 2), für die diversitätsspezifischen Selbstwirksamkeitserwartungen $37,6 \%$ (MZP 2), für die Wichtigkeit alltagsintegrierter Sprachförderung 12,8\% (MZP 2) und für die Wichtigkeit additiver Sprachförderung 3,0\% (MZP 2).

Die Ergebnisse zeigten für den ersten Messzeitpunkt eine positive und signifikante Korrelation zwischen den multikulturellen Überzeugungen und den diversitätsspezifischen Selbstwirksamkeitserwartungen $(\beta=0,48, p<0,01)$, die für den zweiten Messzeitpunkt mit $\beta=0,22(p<0,01)$ allerdings deutlich schwächer ausfiel (Frage- 


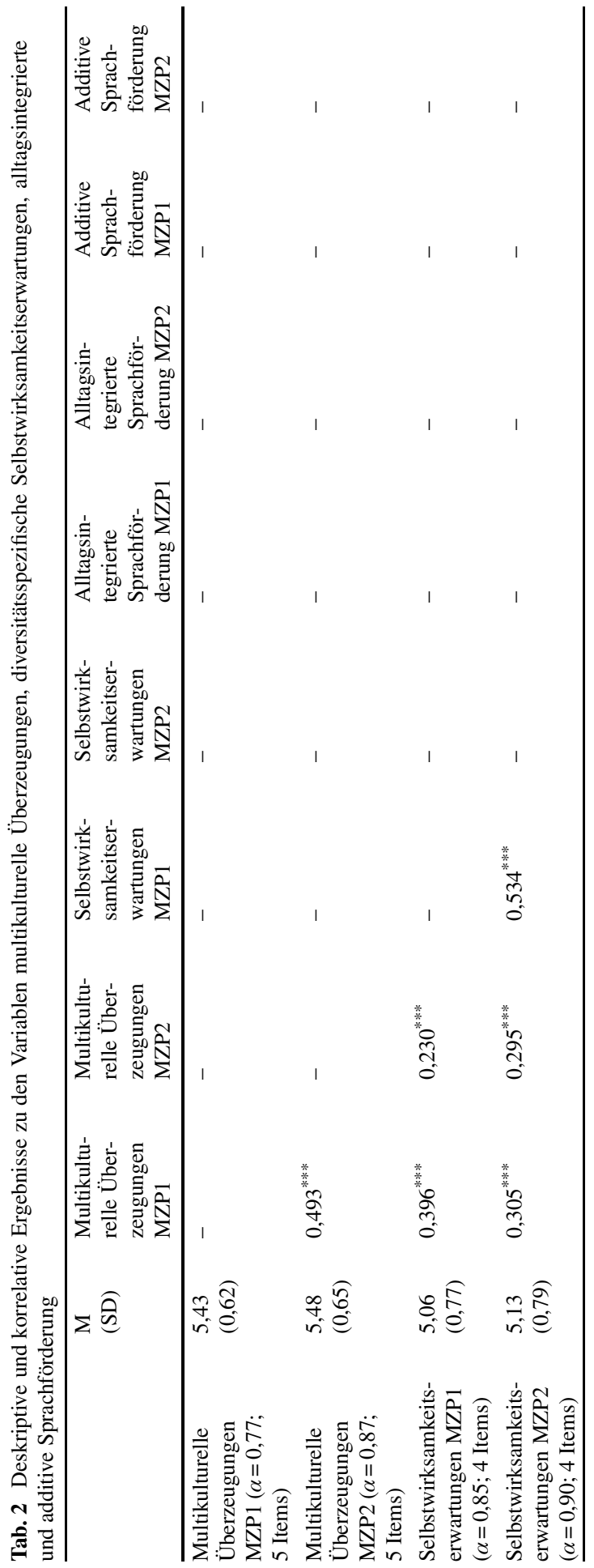




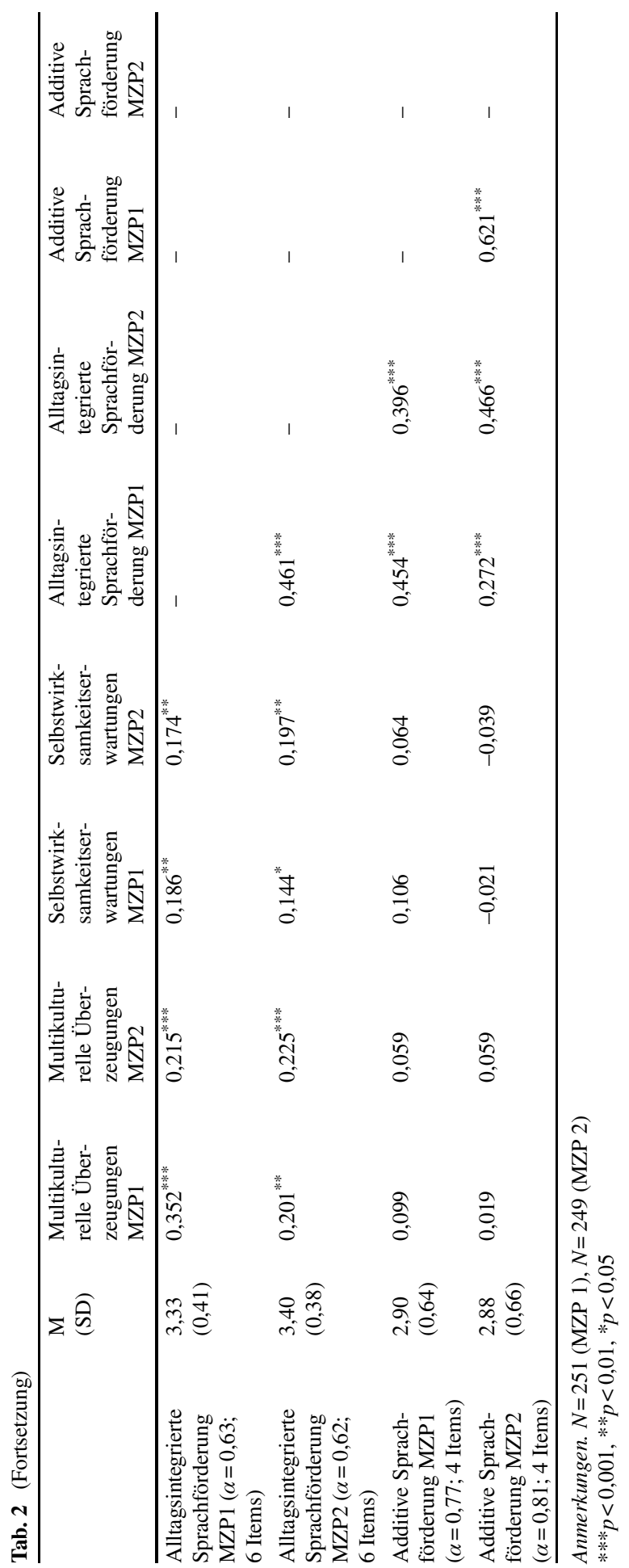




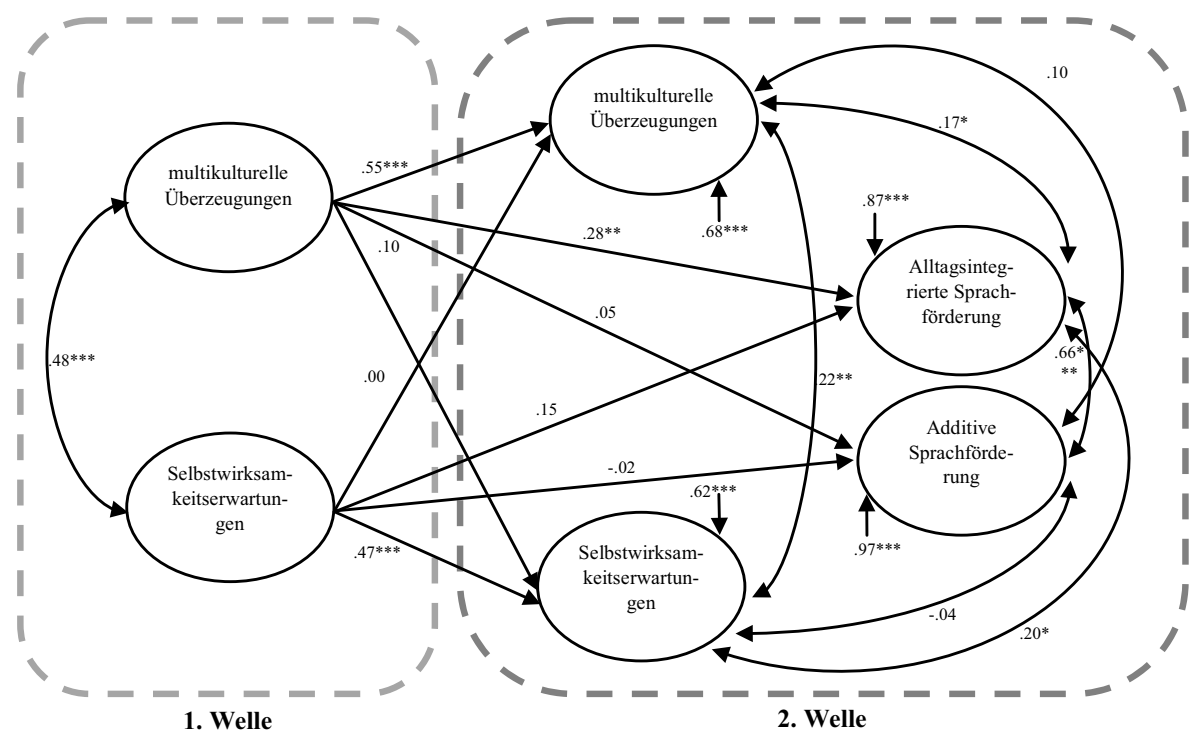

Abb. 1 Strukturgleichungsmodell zum Zusammenhang zwischen multikulturellen Überzeugungen, Selbstwirksamkeitserwartungen und Wichtigkeit sprachpädagogischer Förderstrategien Anmerkung. $\chi^{2}(\mathrm{df})=598,92$ (421); $\mathrm{CFI}=0,94 ; \mathrm{TLI}=0,93 ; \mathrm{RMSEA}=0,04 ; \mathrm{SRMR}=0,05 . * p<0,05 . * * p<0,01$. $* * * p<0,001$

stellung 1a). Im Längsschnitt konnten keine signifikanten Zusammenhänge zwischen den multikulturellen Überzeugungen und den diversitätsspezifischen Selbstwirksamkeitserwartungen festgestellt werden (MÜ1 auf SE2: $\beta=0,10 ; p=0,19$; SE1 auf MÜ2: $\beta=0,00 ; p=0,99)$. Bei den diversitätsspezifischen Selbstwirksamkeitserwartungen konnte zum zweiten Messzeitpunkt allerdings ein signifikanter Zusammenhang mit dem relativen Anteil an Kindern mit Migrationshintergrund in der Einrichtung ermittelt werden $(\beta=0,17 ; p=0,02)$. Die Berufserfahrung im frühpädagogischen Kontext (MÜ2: $\beta=0,70, p=0,27$; SE2: $\beta=0,02, p=0,75$ ), der Migrationshintergrund (MÜ2: $\beta=-0,03, p=0,63$; SE2: $\beta=-0,06, p=0,33$ ) sowie die Ausbildung der Sprachexpertinnen (MÜ2: $\beta=0,08, p=0,22$; SE2: $\beta=-0,04, p=0,54$ ) standen weder in einem signifikanten Zusammenhang zu den multikulturellen Überzeugungen noch zu den diversitätsspezifischen Selbstwirksamkeitserwartungen.

Die autoregressive Beziehung (Fragestellung 1b) zwischen den multikulturellen Überzeugungen vom ersten und zweiten Messzeitpunkt zeigte eine Korrelation von $\beta=0,55(p<0,01)$. Auf Individualebene ließen sich auf Basis der Differenzen zwischen den beiden Messzeitpunkten bei 60,2\% der Sprachexpertinnen keine Veränderung der multikulturellen Überzeugungen konstatieren, bei 21,7\% stiegen sie an und bei $18,1 \%$ nahmen die Überzeugungen ab. Bei den diversitätsspezifischen Selbstwirksamkeitserwartungen deutete die mittlere Korrelation von $\beta=0,47$ $(p<0,01)$ zwischen dem ersten und zweiten Messzeitpunkt auf ein sehr ähnliches Ergebnis hin. Auf Individualebene zeigten sich bei 54,6\% der Sprachexpertinnen keine Veränderungen in den diversitätsspezifischen Selbstwirksamkeitserwartungen, 
bei $25,7 \%$ stiegen sie an und bei $19,7 \%$ nahmen die Selbstwirksamkeitserwartungen $\mathrm{ab}$.

\subsection{Der Zusammenhang zwischen Überzeugungen zum Umgang mit kultureller Diversität und der Wichtigkeit sprachpädagogischer Förderstrategien}

Die multikulturellen Überzeugungen zum ersten Messzeitpunkt waren positiv mit der eingeschätzten Wichtigkeit alltagsintegrierter sprachpädagogischer Förderstrategien zum zweiten Messzeitpunkt assoziiert $(\beta=0,28, p<0,01)$. Für den Zusammenhang zwischen multikulturellen Überzeugungen zum ersten Messzeitpunkt und der Wichtigkeit additiver sprachpädagogischer Förderstrategien zum zweiten Messzeitpunkt konnte jedoch kein signifikanter Effekt festgestellt werden $(\beta=0,05, p=0,57)$ (Fragestellung 2a). Für die diversitätsspezifischen Selbstwirksamkeitserwartungen zum ersten Messzeitpunkt zeigten sich im Vergleich dazu weder für die Wichtigkeit alltagsintegrierter $(\beta=0,15, p=0,15)$ noch für die der additiven sprachpädagogischen Förderstrategien zum zweiten Messzeitpunkt signifikante Zusammenhänge $(\beta=-0,02, p=0,82)$ (Fragestellung 2b). Das Strukturgleichungsmodell weist einen weiteren signifikanten - jedoch negativen - Zusammenhang zwischen dem akademischen Bildungsabschluss und der Wichtigkeit additiver sprachpädagogischer Förderstrategien zum zweiten Messzeitpunkt auf $(\beta=-0,16, p=0,04$; AlS2: $\beta=-0,14$, $p=0,09$ ). Die Berufserfahrung im frühpädagogischen Kontext (A1S2: $\beta=-0,03$, $p=0,69$; AdS2: $\beta=-0,05, p=0,53)$, der Migrationshintergrund der Sprachexpertinnen (AlS2: $\beta=-0,04, p=0,61$; AdS2: $\beta=0,04, p=0,60$ ) sowie der Anteil an Kindern mit Migrationshintergrund in der Einrichtung (AlS2: $\beta=-0,17, p=0,11$; AdS2: $\beta=-0,05, p=0,62$ ) standen in keinem signifikanten Zusammenhang zu den sprachpädagogischen Förderstrategien.

\section{Zusammenfassung und Diskussion}

Aus einer professionstheoretischen Perspektive heraus untersuchte die vorliegende Studie den Zusammenhang zwischen zwei bereichsspezifischen Kompetenzfacetten - den multikulturellen Überzeugungen und den diversitätsspezifischen Selbstwirksamkeitserwartungen - von frühpädagogischen Sprachexpertinnen und deren Bedeutung für die Wichtigkeit zweier sprachpädagogischer Förderstrategien. Die Ergebnisse des Strukturgleichungsmodells deuten darauf hin, dass multikulturelle Überzeugungen und diversitätsspezifische Selbstwirksamkeitserwartungen in Zusammenhang stehende, aber eigenständige Konstrukte sind. So zeigte sich zu beiden Messzeitpunkten eine positive Korrelation, die allerdings im Laufe der Zeit an Stärke verlor. Es ließ sich im Längsschnitt kein Zusammenhang zwischen den multikulturellen Überzeugungen und den diversitätsspezifischen Selbstwirksamkeitserwartungen feststellen. Die autoregressiven Beziehungen über die beiden Messzeitpunkte lassen auf die generelle Veränderbarkeit der multikulturellen Überzeugungen und der diversitätsspezifischen Selbstwirksamkeitserwartungen schließen. Des Weiteren konnte ein signifikanter Zusammenhang zwischen den multikulturellen Überzeugun- 
gen zum ersten Messzeitpunkt und der eingeschätzten Wichtigkeit alltagsintegrierter sprachpädagogischer Förderstrategien zum zweiten Messzeitpunkt nachgewiesen werden. Dieser Effekt blieb für die diversitätsspezifischen Selbstwirksamkeitserwartungen aus.

Vor dem Hintergrund einer zunehmenden sprachlich-kulturellen Diversität in deutschen Kindertageseinrichtungen und den damit einhergehenden sprachpädagogischen Herausforderungen für den Kitaalltag rücken die professionellen Kompetenzen frühpädagogischer Fachkräfte verstärkt in den Fokus des bildungswissenschaftlichen sowie -bildungspolitischen Diskurses. Die in der vorliegenden Studie untersuchten Sprachexpertinnen scheinen die in den Bildungsplänen verankerten Aufgaben zur Integration und multikulturellen Bildung verinnerlicht zu haben. Die deskriptiven Ergebnisse implizieren, dass die untersuchten Fachkräfte es im Allgemeinen als wichtig erachten, auf die unterschiedlichen kulturellen Hintergründe der Kinder mit Migrationshintergrund einzugehen und sich gleichzeitig zutrauen, die Kinder in ihren spezifischen Bedürfnissen zu fördern. Die kulturelle Diversität der Kinder und ihrer Familien wird somit als Ressource verstanden, die unter der Prämisse der Gleichwertigkeit Einzug in den Kitaaltag finden sollte (vgl. Brandes 2009).

Hinsichtlich des Zusammenhangs zwischen den multikulturellen Überzeugungen und diversitätsspezifischen Selbstwirksamkeitserwartungen der Sprachexpertinnen war es für uns wichtig, die Beziehung der beiden Konstrukte über verschiedene Messzeitpunkte zu betrachten, um Aufschluss über mögliche Interdependenzen zu erhalten. Gemäß den theoretischen Erwartungen bilden multikulturelle Überzeugungen und diversitätsspezifische Selbstwirksamkeitserwartungen unterschiedliche Kompetenzfacetten ab, die in einem engen Zusammenhang stehen. Der positive Zusammenhang zwischen den beiden Kompetenzfacetten zum ersten Messzeitpunkt verlor im Laufe der Zeit allerdings deutlich an Stärke. Es wäre denkbar, dass im Zuge des verstärkten interethnischen Kontaktes mit den Kindern und Familien mit Migrationshintergrund und den damit potenziell einhergehenden kulturellen Differenzen die Überzeugungen und Selbstwirksamkeitserwartungen der Sprachexpertinnen auf unterschiedliche Weise beeinflusst werden (vgl. Bender-Szymanski 2000). Als ein Indiz dafür lässt sich der positive Zusammenhang zwischen dem relativen Anteil an Kindern mit Migrationshintergrund in der Einrichtung und den diversitätsspezifischen Selbstwirksamkeitserwartungen zum zweiten Messzeitpunkt deuten. Der interethische Kontakt kann somit positive Effekte auf die motivationalen Orientierungen gegenüber Migrantengruppen bewirken (vgl. Pettigrew 1998). Die Sprachexpertinnen könnten einen selbstsicheren Habitus entwickelt haben, der ihnen den Umgang mit Kindern und Familien mit Migrationshintergrund erleichtert.

Die autoregressiven Beziehungen der beiden Konstrukte zeigten über den zeitlichen Verlauf Veränderungen auf Individualebene. Tendenziell nahmen sowohl die multikulturellen Überzeugungen als auch die diversitätsspezifischen Selbstwirksamkeitserwartungen eher zu. Dieser Befund steht im Einklang mit Forschungserkenntnissen, die ebenfalls die Veränderbarkeit von Überzeugungen und Selbstwirksamkeitserwartungen belegen konnten (Bender-Szymanski 2000; Kopp 2009). Substantielle Veränderungen scheinen jedoch an Bedingungen geknüpft zu sein. Als entscheidende Bedingungen lassen sich neben den theoretisch vermittelten Inhalten insbesondere die Möglichkeiten zur Reflexion der Person über sich selbst und das 
eigene Verhalten identifizieren (Civitillo et al. 2016b). Nur durch die mentale Betrachtung der eigenen Gefühle und Gedanken gewinnt die Fachkraft Aufschluss über ihren eigenen Anteil an den entstandenen Konflikten, die aus ihrem affektiven und unreflektierten kulturgebundenen Handeln in interkulturellen Interaktionen resultieren können. Die untersuchten Sprachexpertinnen nahmen regelmäßig an Fortbildungen teil, die allerdings primär den Bereich der alltagsintegrierten sprachlichen Bildungsarbeit behandelten. Zusätzlich hatten sie im Rahmen ihrer Tätigkeit die Möglichkeit, zusammen mit dem gesamten Team der Einrichtung an Supervisionen teilzunehmen (BMFSFJ 2015). Inwieweit diese von den Sprachexpertinnen in den einzelnen Einrichtungen zur Reflexion des eigenen Verhaltens genutzt wurden, lässt sich abschließend nicht überprüfen.

Entsprechend der theoretischen Annahmen können Überzeugungen von frühpädagogischen Fachkräften handlungsleitende Implikationen für den Umgang mit Kindern in Kindertageseinrichtungen haben. Die Ergebnisse der vorliegenden Studie verdeutlichen, dass die multikulturellen Überzeugungen und die diversitätsspezifischen Selbstwirksamkeitserwartungen der von uns untersuchten Sprachexpertinnen auf unterschiedliche Weise mit der Wichtigkeit sprachpädagogischer Förderstrategien im Umgang mit kulturell diversen Gruppen in Kindertageseinrichtungen assoziiert sind. Sprachexpertinnen mit ausgeprägten multikulturellen Überzeugungen erachten demnach eine alltagsintegrierte sprachpädagogische Förderung als das effektivere Vorgehen, um insbesondere Kinder mit Migrationshintergrund - bei denen im Vergleich zu deutschen Kindern häufiger ein Rückstand der Deutschsprachkenntnisse zu beobachten ist - in ihrer sprachlichen Entwicklung zu unterstützen. Die Ergebnisse könnten in die Diskussion um alltagsintegrierte und additive Sprachfördermaßnahmen eingebunden werden, sofern zukünftige Forschungsergebnisse Aussagen über die Wirksamkeit zulassen (vgl. Egert und Hopf 2016). In diesem Zusammenhang wäre auch der Einbezug unterschiedlicher Erstsprachen der Kinder zu berücksichtigen. Aktuelle Befunde konnten aufzeigen, dass sich ein erfolgreicher Erstspracherwerb positiv auf den Zweitspracherwerb auswirken kann (Edele und Stanat 2016). Für den frühkindlichen Bereich lassen sich allerdings anhand der derzeitigen Forschungslage weder für die Erstsprachenförderung noch für die frühe bilinguale Sprachförderung fundierte Aussagen treffen. Es existieren sowohl Studien, nach denen eine bilinguale Sprachförderung von Kindern im Vorschulalter einen positiven Effekt auf den kompetenten Erwerb beider Sprachen hat (Farver et al. 2009), als auch Untersuchungen, die nahe legen, dass eine Bevorzugung der Erstsprache im Umgang mit mehrsprachigen Kindern ein weniger elaboriertes und kindgerechtes Sprachverhalten der Erzieherinnen bedingt (Kratzmann et al. 2013). Unabhängig von der Frage, in welchem Maße die Erstsprache von Kindern mit Migrationshintergrund in Kindertageseinrichtungen letztlich Eingang finden soll, ist es für bilingual aufwachsende Kinder wichtig, dass sich Fachkräfte mit den typischen Herausforderungen beim Zweitspracherwerb auseinandersetzen. Schließlich ermöglicht der bilinguale Sprachgebrauch eine förderliche Identitätsbildung beim Heranwachsen der Kinder (Stitzinger und Lüdtke 2014). Hier spielen die Überzeugungen im Umgang mit kultureller Diversität eine entscheidende Rolle. Die Vermeidung von Bewertungshierarchien beim alltäglichen Sprachgebrauch in Kindertageseinrichtungen ist insbesondere bei denjenigen Familiensprachen zentral, die nicht den gängigen 
Verkehrs- bzw. Bildungssprachen angehören und somit weniger Prestige in Form emotionalen und kulturellen Wertes genießen (Frigerio Sayilir 2007). Die multikulturellen Überzeugungen von frühpädagogischen Fachkräften können unter der regulativen Prämisse, Erziehungs- und Bildungsangebote bereitzustellen, von denen alle Kinder profitieren können, einen wichtigen Beitrag für eine positive Integration von Kindern mit Migrationshintergrund leisten. Daraus wird unmittelbar ersichtlich, dass Kindertageseinrichtungen für Kinder mit Migrationshintergrund entscheidende Wegbereiter zur Sprachförderung im Deutschen und damit auch für die aktive Teilnahme am gesellschaftlichen Leben sein können.

\subsection{Limitationen}

Die vorliegenden Untersuchungen wurden anhand einer besonderen Stichprobe von speziell ausgebildeten pädagogischen Fachkräften durchgeführt, die sich im Vergleich zu den Beschäftigten in der Frühen Bildung durch einen überdurchschnittlich hohen Anteil an Hochschulabsolventinnen zusammensetzt (Autorengruppe Fachkräftebarometer und Deutsches Jugendinstitut 2017). Studien deuten darauf hin, dass ein höheres Bildungsniveau mit einer stärkeren Akzeptanz von Menschen anderer kultureller Herkunft einhergeht (van de Vijver et al. 2008). Die Besonderheiten der Stichprobe liegen neben dem hohen Professionalisierungsgrad auch darin, dass die Fachkräfte fortlaufend über mehrere Jahre in der sprachpädagogischen Bildungsarbeit weiterqualifiziert wurden. Die ausgeprägten multikulturellen Überzeugungen und diversitätsspezifischen Selbstwirksamkeitserwartungen könnten daher durch drei Effekte erklärt werden: Einerseits kann von einer positiven Selektion aus dem pädagogischen Feld ausgegangen werden. Da das Bundesprogramm ,Schwerpunkt-Kitas Sprache \& Integration“ als ein grundsätzliches Ziel die Unterstützung von Kindern aus Familien mit Migrationshintergrund definiert hatte, fühlten sich wahrscheinlich eher Fachkräfte angesprochen, die von vornherein positive Überzeugungen gegenüber dieser Gruppe aufwiesen.

Andererseits könnten die günstigen Überzeugungen ebenfalls Effekte des Bundesprogramms widerspiegeln, da die Erhebung zum ersten Messzeitpunkt erst nach mehr als eineinhalb Jahren Programmlaufzeit erfolgte. Dazu korrespondieren die deskriptiven Befunde zu der von den Sprachexpertinnen eingeschätzten Wichtigkeit sprachpädagogischer Förderstrategien. Da das Bundesprogramm ,Schwerpunkt-Kitas Sprache \& Integration“ einen nachhaltigen Anstoß zur Implementierung alltagsintegrierter sprachlicher Bildung in den Kindertageseinrichtungen geben wollte, spiegelt sich diese Gewichtung zwischen den beiden unterschiedlichen sprachpädagogischen Ansätzen vermutlich auch in den Überzeugungen der Fachkräfte wider. Alltagsintegrierte Förderung wird als das effektivere Vorgehen im frühkindlichen Bildungsbereich angesehen, um insbesondere Kindern mit einem Sprachrückstand im Deutschen korrekte Sprachvorbilder zu gewährleisten und eine sprachanregende Umgebung zu schaffen (Buschmann und Joos 2011). Das Ziel dieses Bundesprogramms - alltagsintegrierte sprachliche Bildung in der Kindertagesbetreuung zu verankern - scheint somit gelungen zu sein.

Ferner lassen sich anhand der Datenlage Effekte der sozialen Erwünschtheit nicht ausschließen. Selbsteinschätzungen bleiben immer subjektiv und unterliegen tenden- 
ziell einer Über- oder Unterschätzung der eigenen Kompetenzfacetten (Settlage et al. 2009; Neugebauer 2010). Diese Tendenz könnte durch die Tatsache verstärkt worden sein, dass der angemessene Umgang mit kultureller Diversität ein durch bildungspolitische Diskussionen stark normativ aufgeladenes Thema darstellt. Ebenso werden im öffentlichen Bewusstsein sprachpädagogische Förderstrategien weitgehend befürwortet. Diese Annahme könnte insbesondere für das Konzept der alltagsintegrierten Sprachförderung gelten, das derzeit insbesondere von Praktizierenden weithin akzeptiert und vertreten wird. Künftige Forschung sollte daher an der Weiterentwicklung der Items zu den sprachpädagogischen Förderstrategien arbeiten, um die Varianz in den Antworten zu erhöhen. Zudem sind einem die eigenen Überzeugungen nicht immer bewusst und bisweilen auch nicht immer direkt zugänglich (Kunter und Trautwein 2013). Folglich sollten zusätzlich indirekte Maße zur Erfassung von Überzeugungen berücksichtigen werden, um neben den potenziellen Unterschieden zwischen impliziten und expliziten Überzeugungen auch differenzielle Effekte auf die Wichtigkeit sprachpädagogischer Förderstrategien aufdecken zu können. Eine Metaanalyse von Hofmann et al. (2005) konnte jedoch positive Zusammenhänge und eine substanzielle Überlappung zwischen impliziten und expliziten Maßen zur Erfassung von Einstellungskomponenten aufzeigen.

Als eine weitere Einschränkung der Studie ist zu nennen, dass die selbsteingeschätzte Wichtigkeit alltagsintegrierter und additiver sprachpädagogischer Förderung nur ein vager Indikator für die tatsächlich stattfindende sprachliche Interaktion zwischen den Sprachexpertinnen und den Kindern ist. Obwohl die unterschiedliche Gewichtung der beiden Ansätze einen Hinweis auf die Kommunikationsstrategien der Sprachexpertinnen liefert, können verschiedene Umstände dazu führen, dass Sprachexpertinnen nicht gemäß ihren sprachpädagogischen Überzeugungen handeln. Unter diesen Umständen ist beispielsweise die emotionale Steuerungskomponente aufzuführen, die das Handeln stets begleitet. Es muss an dieser Stelle letztlich offenbleiben, ob und, wenn ja, inwieweit die Sprachexpertinnen auf der Performanzebene sowohl einen kultursensitiven Umgang als auch alltagsintegrierte sprachpädagogische Förderung mit den Kindern pflegen.

\subsection{Implikationen für die Ausbildung}

Eine qualitativ hochwertige frühkindliche Bildung und Erziehung räumt - insbesondere unter dem Aspekt der Sicherung von Chancengerechtigkeit - der sprachpädagogischen Förderung einen zentralen Stellenwert ein (Jugendministerkonferenz (JMK) \& Kultusministerkonferenz (KMK) 2004). Studien weisen darauf hin, dass vor allem Kinder mit Migrationshintergrund hinsichtlich ihrer Sprachkompetenzen von einer längeren vorschulischen Betreuung profitieren (Burchinal et al. 2000; Spiess et al. 2003). Gleichzeitig sollten frühpädagogische Fachkräfte entsprechend auf die Herausforderungen vorbereitet werden, die mit der steigenden kulturellen Diversität in deutschen Kindertageseinrichtungen einhergehen. Die Ergebnisse der vorliegenden Studie deuten auf die Bedeutung von multikulturellen Überzeugungen für die alltagsintegrierte sprachpädagogische Förderung in kulturell diversen Kindertageseinrichtungen hin. Alltagsintegrierte sprachliche Förderung könnte gerade für Kinder mit Migrationshintergrund geeignete Voraussetzungen für eine anregende 
und kultursensible Sprachförderung bieten, da durch interessengeleitete Alltagsaktivitäten individuell auf den Sprachentwicklungsstand der Kinder eingegangen und auch Bezug zur Familiensprache genommen werden kann. Dazu müssen die sprachförderlichen Potenziale des Kitaalltags von den Fachkräften allerdings auch genutzt werden, was häufig noch nicht der Fall ist (Girolametto et al. 2006). Zusätzlich bedarf es einer systematischen Abstimmung mit wirkungsvollen additiven Sprachförderkonzepten, damit alle Kinder vor dem Schuleintritt über bildungsrelevante Sprachkompetenzen verfügen. Die Befunde der vorliegenden Studie geben weitere Hinweise für die in Professionalisierungstheorien beschriebenen handlungsleitenden Implikationen von Überzeugungen für das pädagogische Alltagsgeschehen. Multikulturelle Überzeugungen können demnach einen bedeutenden Beitrag für ein differenziertes, vorurteilsfreies und wertschätzendes Verständnis ethnisch-kultureller Diversität in Kindertageseinrichtungen leisten. Die Förderung multikultureller Überzeugungen von frühpädagogischen Fachkräften scheint gerade vor dem Hintergrund von Befunden zentral, nach denen sich ein besonders hoher Anteil an Kindern mit Migrationshintergrund - auch bei günstigen Rahmenbedingungen - negativ auf die Anregungsqualität in Kindertageseinrichtungen auswirkt (Kuger und Kluczniok 2009).

Für die Aus- und Weiterbildung frühpädagogischer Fachkräfte implizieren die Befunde, dass sich diese auch auf deren Überzeugungen zu kultureller Diversität und Sprachförderung konzentrieren sollten. Die Bildung von neuen Überzeugungen ist im Vergleich zur Vermittlung von Fachwissen oder der Erweiterung von Fähigkeiten ein längerfristiger Prozess und erfordert sehr viel mehr Intensität. Da sich pädagogische Fachkräfte durch eine hohe Weiterbildungsquote von über $80 \%$ auszeichnen (Buschle und Gruber 2018), könnten Fort- und Weiterbildungen langfristig und aufeinander aufbauend dazu genutzt werden, um einerseits den notwendigen Raum für Praxisreflexion bzw. Supervision zu schaffen und andererseits Wissen hinsichtlich typischer Herausforderungen von Kindern und Familien mit Migrationshintergrund (z.B. in Bezug auf Mehrsprachigkeit) und den konstruktiven Umgang mit ihnen zu vermitteln. Die Veränderbarkeit über die Zeit spricht gleichwohl dafür, dass diese Überzeugungssysteme auch durch andere externe Reize, wie beispielsweise Fort- und Weiterbildungen ansprechbar sind. In Zeiten zunehmender kultureller Diversität im Primar- und Sekundarbereich könnten die Ergebnisse der vorliegenden Studie ferner ein Indiz dafür sein, auch in der Lehrerausbildung die Übernahme von multikulturellen Überzeugungen stärker einzubeziehen. Praxisnahe Weiterbildungen können die Sensibilität gegenüber den eigenen Vorurteilen und vorschnellen Verallgemeinerungen schärfen, wodurch die Fachkraft sowohl Hinweise auf die eigenen „,blinden Flecken“ als auch auf das pädagogisch angemessene Verhalten erhält. Die Übersichtsarbeit von Civitillo et al. (2016b) verdeutlicht, dass die Kombination aus Seminaren, Felderfahrung und der Möglichkeit zur Selbstreflexion die bislang wirksamste Methode zur positiven Beeinflussung von Überzeugungen angehender Lehrkräfte hinsichtlich kultureller Diversität darstellt. Eine wertschätzende Haltung pädagogischer Fachkräfte gegenüber sprachlich-kultureller Diversität in Kindertageseinrichtungen kann als eine zentrale Voraussetzung für die erfolgreiche Integration aller Kinder in eine multikulturelle Gesellschaft angesehen werden. In Verbindung mit der Implementation sprachpädagogischer Fördermaßnahmen könn- 
ten so herkunftsbedingte Ungleichheiten bereits in einer frühen Phase abgeschwächt werden.

Funding Open Access funding provided by Projekt DEAL.

Open Access Dieser Artikel wird unter der Creative Commons Namensnennung 4.0 International Lizenz veröffentlicht, welche die Nutzung, Vervielfältigung, Bearbeitung, Verbreitung und Wiedergabe in jeglichem Medium und Format erlaubt, sofern Sie den/die ursprünglichen Autor(en) und die Quelle ordnungsgemäß nennen, einen Link zur Creative Commons Lizenz beifügen und angeben, ob Änderungen vorgenommen wurden.

Die in diesem Artikel enthaltenen Bilder und sonstiges Drittmaterial unterliegen ebenfalls der genannten Creative Commons Lizenz, sofern sich aus der Abbildungslegende nichts anderes ergibt. Sofern das betreffende Material nicht unter der genannten Creative Commons Lizenz steht und die betreffende Handlung nicht nach gesetzlichen Vorschriften erlaubt ist, ist für die oben aufgeführten Weiterverwendungen des Materials die Einwilligung des jeweiligen Rechteinhabers einzuholen.

Weitere Details zur Lizenz entnehmen Sie bitte der Lizenzinformation auf http://creativecommons.org/ licenses/by/4.0/deed.de.

\section{Literatur}

Abelson, R. P. (1979). Differences between belief and knowledge systems. Cognitive Science, 3, 355-366. https://doi.org/10.1207/s15516709cog0304_4.

Anders, Y. (2012). Modelle professioneller Kompetenzen für frühpädagogische Fachkräfte. Aktueller Stand und ihr Bezug zur Professionalisierung. Expertise zum Gutachten „Professionalisierung in der Frühpädagogik“ im Auftrag des Aktionsrats Bildung. München: vbm.

Anders, Y. (2013). Stichwort: Auswirkungen frühkindlicher institutioneller Betreuung und Bildung. Zeitschrift für Erziehungswissenschaft, 16(2), 237-275. https://doi.org/10.1007/s11618-013-0357-5.

Anders, Y., Ballaschk, I., Dietrichkeit, T., Flöter, M., Groeneveld, I., Lee, H.-J., \& Nattefort, R. R. (2016). Implementation und Auswirkungen des Bundesprogramms ,,Schwerpunkt-Kitas Sprache \& Integration": Studie und Ergebnisse im Detail. In H.-G. Roßbach, Y. Anders \& W. Tietze (Hrsg.), Wissenschaftliche Evaluation des Bundesprogramms „Schwerpunkt-Kitas Sprache \& Integration“. Bamberg/Berlin: Otto-Friedrich-Universität Bamberg/Freie Universität Berlin.

Autorengruppe Bildungsberichterstattung (2018). Bildung in Deutschland 2018. Ein indikatorengestützter Bericht mit einer Analyse zu Bildung und Migration. Bielefeld: wbv. https://doi.org/10.3278/ $6001820 \mathrm{fw}$.

Autorengruppe Fachkräftebarometer, \& Deutsches Jugendinstitut (2017). Fachkräftebarometer Frühe Bildung 2017. München: Deutsches Jugendinstitut.

Bandura, A. (1997). Self-efficacy. The exercise of control. New York, NY: Freeman.

Bandura, A. (2001). Social cognitive theory: an agentic perspective. Annual review of psychology, 52, 1-26. https://doi.org/10.1146/annurev.psych.52.1.1.

Becker, B. (2006). Der Einfluss des Kindergartens als Kontext zum Erwerb der deutschen Sprache bei Migrantenkindern. Zeitschrift für Soziologie, 35, 449-464. https://doi.org/10.1515/zfsoz-2006-0602.

Becker, B. (2010). Ethnische Unterschiede bei der Kindergartenselektion: Die Wahl von unterschiedlich stark segregierten Kindergärten in deutschen und türkischen Familien. In B. Becker \& D. Reimer (Hrsg.), Vom Kindergarten bis zur Hochschule (S. 17-47). Wiesbaden: VS. https://doi.org/10.1007/ 978-3-531-92105-1_2.

Bender-Szymanski, D. (2000). Learning through cultural conflict? A longitudinal analysis of German teachers' strategies for coping with cultural diversity at school. European Journal of Teacher Education, 23, 229-250. https://doi.org/10.1080/02619760120049120.

Biedinger, N., \& Becker, B. (2010). Frühe ethnische Bildungsungleichheit: Der Einfluss des Kindergartenbesuchs auf die deutsche Sprachfähigkeit und die allgemeine Entwicklung. In B. Becker \& D. Reimer (Hrsg.), Vom Kindergarten bis zur Hochschule (S. 49-79). Wiesbaden: VS. https://doi.org/10.1007/ 978-3-531-92105-1_3. 
Blossfeld, H.-P. (2012). Professionalisierung in der Frühpädagogik. Qualifikationsniveau und -bedingungen des Personals in Kindertagesstätten (1. Aufl.). Münster: Waxmann.

Brandes, H. (2009). Möglichkeiten und Grenzen interkultureller Arbeit in Kindertageseinrichtungen. In M. Spetsmann-Kunkel (Hrsg.), Gegen den Mainstream. Kritische Perspektiven auf Bildung und Gesellschaft; Festschrift für Georg Hansen (Lernen für Europa, Bd. 13, S. 80-93). Münster: Waxmann.

Brandes, H., Friedel, S., \& Röseler, W. (2011). Gleiche Startchancen schaffen! Bildungsbenachteiligung und Kompensationsmöglichkeiten in Kindergärten; eine repräsentative Erhebung in Sachsen (Dresdner Beiträge zu Bildung und Erziehung, Bd. 1). Opladen: Budrich UniPress.

Bryan, L. A., \& Atwater, M. M. (2002). Teacher beliefs and cultural models: a challenge for science teacher preparation programs. Science Education, 86, 821-839. https://doi.org/10.1002/sce.10043.

Bundesministerium für Familie, Senioren, Frauen und Jugend (2005). Zwölfter Kinder- und Jugendbericht: „Bildung, Betreuung und Erziehung vor und neben der Schule“ - Bericht über die Lebenssituation junger Menschen und die Leistungen der Kinder- und Jugendhilfe in Deutschland. Berlin: Bundesministerium für Familie, Senioren, Frauen und Jugend.

Bundesministerium für Familie, Senioren, Frauen und Jugend (2008). Nationaler Aktionsplan. Für ein kindgerechtes Deutschland 2005-2010 (Zwischenbericht). Berlin: Bundesministerium für Familie, Senioren, Frauen und Jugend.

Bundesministerium für Familie, Senioren, Frauen und Jugend (2015). Abschlussbericht zum Bundesprogramm „Schwerpunkt-Kitas Sprache \& Integration“

Burchinal, M.R., Peisner-Feinberg, E., Bryant, D. M., \& Clifford, R. (2000). Children's social and cognitive development and child-care quality: testing for differential associations related to poverty, gender, or ethnicity. Applied Developmental Science, 4, 149-165. https://doi.org/10.1207/ S1532480XADS0403_4.

Burns, M. O., \& Seligman, M.E. (1989). Explanatory style across the life span: evidence for stability over 52 years. Journal of personality and social psychology, 56, 471-477.

Buschle, C., \& Gruber, V. (2018). Die Bedeutung von Weiterbildung für das Arbeitsfeld Kindertageseinrichtung (WiFF Studien, Bd. 30). München: Weiterbildungsinitiative Frühpädagogische Fachkräfte.

Buschmann, A., \& Joos, B. (2011). Alltagsintegrierte Sprachförderung in der Kinderkrippe - Effektivität eines sprach-basierten Interaktionstrainings für pädagogisches Fachpersonal. Verhaltenstherapie \& psychosoziale Praxis, 43, 303-312.

Civitillo, S., Juang, L., Schachner, M. K., \& Börnert, M. (2016a). Validierung einer deutschen Version der „Culturally Responsive Classroom Management Self-Efficacy Scale“. Empirische Sonderpädagogik, 3, 279-288.

Civitillo, S., Juang, L., \& Schachner, M. (2016b). Kulturelle Vorstellungen in der Lehrerbildung: Status quo und Möglichkeiten der Veränderung.

Derman-Sparks, L., \& Ramsey, P.G. (2006). What if all the kids are white? Anti-bias multicultural education with young children and families (Early childhood education series). New York, NY: Teachers College Press.

Dickinson, D. K., \& Porche, M. V. (2011). Relation between language experiences in preschool classrooms and children's kindergarten and fourth-grade language and reading abilities. Child development, 82, 870-886. https://doi.org/10.1111/j.1467-8624.2011.01576.x.

Ebert, S., Lockl, K., Weinert, S., Anders, Y., Kluczniok, K., \& Rossbach, H.-G. (2013). Internal and external influences on vocabulary development in preschool children. School Effectiveness and School Improvement, 24, 138-154. https://doi.org/10.1080/09243453.2012.749791.

Edele, A., \& Stanat, P. (2016). The role of first-language listening comprehension in second-language reading comprehension. Journal of Educational Psychology, 108, 163-180.

Egert, F., \& Hopf, M. (2016). Zur Wirksamkeit von Sprachförderung in Kindertageseinrichtungen in Deutschland. Kindheit und Entwicklung, 25, 153-163. https://doi.org/10.1026/0942-5403/a000199.

Farver, J. A. M., Lonigan, C. J., \& Eppe, S. (2009). Effective early literacy skill development for young Spanish-speaking English language learners: an experimental study of two methods. Child development, 80, 703-719. https://doi.org/10.1111/j.1467-8624.2009.01292.x.

Fischer, M. Y., \& Pfost, M. (2015). Wie effektiv sind Maßnahmen zur Förderung der phonologischen Bewusstheit? Zeitschrift für Entwicklungspsychologie und Pädagogische Psychologie, 47, 35-51. https://doi.org/10.1026/0049-8637/a000121.

Fives, H., \& Buehl, M. M. (2012). Spring cleaning for the "messy" construct of teachers' beliefs: What are they? Which have been examined? What can they tell us? In K. R. Harris (Hrsg.), APA educational psychology handbook. Volume 2: Individual differences and cultural and contextual factors (2. Aufl, APA handbooks in psychology, S. 471-499). Washington, D.C.: American Psychological Association. https://doi.org/10.1037/13274-019. 
Frigerio Sayilir, C. (2007). Zweisprachig aufwachsen - zweisprachig sein. Der Erwerb zweier Erstsprachen aus der handlungstheoretischen Sicht der Kooperativen Pädagogik (Internationale Hochschulschriften, Bd. 490). Freiburg (CH): Zugl.

Fröhlich-Gildhoff, K., Nentwig-Gesemann, I., \& Pietsch, S. (2011). Kompetenzorientierung in der Qualifizierung frühpädagogischer Fachkräfte. Eine Expertise der Weiterbildungsinitiative Frühpädagogische Fachkräfte (WiFF) (Ausbildung, Bd. 19). München: Dt. Jugendinst.

Gagarina, N., Posse, D., Düsterhöft, S., Topaj, N., \& Acikgöz, D. (2014). Sprachförderung bei Mehrsprachigkeit: Erste Ergebnisse der BIVEM-Studie zur Wirksamkeit von Sprachförderung bei jüngeren mehrsprachigen Kindern: Eine Studie des Berliner Interdisziplinären Verbundes für Mehrsprachigkeit (BIVEM). Spektrum Patholinguistik, 7, 139-148.

Gay, G. (2010). Acting on beliefs in teacher education for cultural diversity. Journal of Teacher Education, 61, 143-152. https://doi.org/10.1177/0022487109347320.

Girolametto, L., Weitzman, E., \& Greenberg, J. (2006). Facilitating language skills - in-service education for early childhood educators and preschool teachers. Infants \& Young Children, 19, 36-49.

Göbel, K., \& Helmke, A. (2010). Intercultural learning in English as foreign language instruction: the importance of teachers' intercultural experience and the usefulness of precise instructional directives. Teaching and Teacher Education, 26, 1571-1582. https://doi.org/10.1016/j.tate.2010.05.008.

Göbel, K., \& Hesse, H.-G. (2008). Vermittlung interkultureller Kompetenzen im Englischunterricht. In DESI-Konsortium (Hrsg.), Unterricht und Kompetenzerwerb in Deutsch und Englisch. Ergebnisse der DESI-Studie (S. 398-410). Weinheim: Beltz.

Gogolin, I. (2016). Multikulturalität als Herausforderung. In H. Faulstich-Wieland, H.-U. Grunder, K. Kansteiner \& H. Moser (Hrsg.), Umgang mit Heterogenität und Differenz. (S. 49-72). Baltmannsweiler: Schneider Verlag Hohengehren.

Guo, Y., Piasta, S.B., Justice, L. M., \& Kaderavek, J.N. (2010). Relations among preschool teachers' self-efficacy, classroom quality, and children's language and literacy gains. Teaching and Teacher Education, 26, 1094-1103. https://doi.org/10.1016/j.tate.2009.11.005.

Hachfeld, A., Hahn, A., Schroeder, S., Anders, Y., Stanat, P., \& Kunter, M. (2011). Assessing teachers' multicultural and egalitarian beliefs: the Teacher Cultural Beliefs Scale. Teaching and Teacher Education, 27, 986-996. https://doi.org/10.1016/j.tate.2011.04.006.

Hachfeld, A., Hahn, A., Schroeder, S., Anders, Y., \& Kunter, M. (2015). Should teachers be colorblind? How multicultural and egalitarian beliefs differentially relate to aspects of teachers' professional competence for teaching in diverse classrooms. Teaching and Teacher Education, 48, 44-55. https:// doi.org/10.1016/j.tate.2015.02.001.

Hachfeld, A., Schroeder, S., Anders, Y., Hahn, A., \& Kunter, M. (2012). Multikulturelle Überzeugungen. Zeitschrift für Pädagogische Psychologie, 26, 101-120. https://doi.org/10.1024/1010-0652/a000064.

Hasselhorn, M., \& Sallat, S. (2014). Sprachförderung zur Prävention von Bildungsmisserfolg. In S. Sallat, M. Spreer \& C. W. Glück (Hrsg.), Sprache professionell fördern. Kompetent, vernetzt, innovativ (1. Aufl., Sprachheilpädagogik aktuell, Bd. 1, S. 28-39). Idstein: Schulz-Kirchner.

Hofmann, N., Polotzek, S., Roos, J., \& Schöler, H. (2008). Sprachförderung im Vorschulalter - Evaluation dreier Sprachförderkonzepte. Diskurs Kindheits- und Jugendforschung, 3, 291-300.

Hofmann, W., Gawronski, B., Gschwendner, T., Le, H., \& Schmitt, M. (2005). A meta-analysis on the correlation between the implicit association test and explicit self-report measures. Personality \& social psychology bulletin, 31, 1369-1385. https://doi.org/10.1177/0146167205275613.

Hu, L.-T., \& Bentler, P. M. (1999). Cutoff criteria for fit indexes in covariance structure analysis: conventional criteria versus new alternatives. Structural Equation Modeling: A Multidisciplinary Journal, 6, 1-55. https://doi.org/10.1080/10705519909540118.

Jampert, K. (2002). Schlüsselsituation Sprache. Spracherwerb im Kindergarten unter besonderer Berücksichtigung des Spracherwerbs bei mehrsprachigen Kindern. Diskurs, 10, 60-68. https://doi.org/10. 1007/978-3-322-89819-7.

Jerusalem, M., \& Hopf, D. (Hrsg.). (2002). Selbstwirksamkeit und Motivationsprozesse in Bildungsinstitutionen (Zeitschrift für Pädagogik, Beiheft: 44). Weinheim: Beltz.

Jugendministerkonferenz (JMK), \& Kultusministerkonferenz (2004). Gemeinsamer Rahmen der Länder für die frühe Bildung in Kindertageseinrichtungen. https://www.kmk.org/fileadmin/Dateien/ veroeffentlichungen_beschluesse/2004/2004_06_03-Fruehe-Bildung-Kindertageseinrichtungen.pdf. Zugegriffen: 13. Nov. 2018.

Jungmann, T., Koch, K., \& Etzien, M. (2013). Effektivität alltagsintegrierter Sprachförderung bei ein- und zwei- bzw. mehrsprachig aufwachsenden Vorschulkindern. Frühe Bildung, 2, 110-121. https://doi. org/10.1026/2191-9186/a000098. 
Justice, L. M., Mashburn, A., Hamre, B., \& Pianta, R. (2008). Quality of language and literacy instruction in preschool classrooms serving at-risk pupils. Early childhood research quarterly, 23, 51-68. https:// doi.org/10.1016/j.ecresq.2007.09.004.

Kammermeyer, G., \& Roux, S. (2013). Sprachbildung und Sprachförderung. In M. Stamm \& D. Edelmann (Hrsg.), Handbuch frühkindliche Bildungsforschung (S. 515-528). Wiesbaden: Springer VS.

Kempert, S., Edele, A., Rauch, D., Wolf, K. M., Paetsch, J., \& Darsow, A. (2016). Die Rolle der Sprache für zuwanderungsbezogene Ungleichheiten im Bildungserfolg. In C. Diehl, C. Hunkler \& C. Kristen (Hrsg.), Ethnische Ungleichheiten im Bildungsverlauf (Bd. 80, S. 157-241). Wiesbaden: Springer. https://doi.org/10.1007/978-3-658-04322-3_5.

Kluczniok, K., Anders, Y., \& Ebert, S. (2011). Fördereinstellungen von Erzieherinnen. Einflüsse auf die Gestaltung von Lerngelegenheiten im Kindergarten und die kindliche Entwicklung früher numerischer Kompetenzen. Frühe Bildung. https://doi.org/10.1026/2191-9186/a000002.

Kopp, B. (2009). Inklusive Überzeugungen und Selbstwirksamkeit im Umgang mit Heterogenität - Wie denken Studierende des Lehramts für Grundschulen? Empirische Sonderpädagogik, 1, 5-25.

Kratzmann, J., Jahreiß, S., Frank, M., Ertanir, B., \& Sachse, S. (2017). Einstellungen pädagogischer Fachkräfte in Kindertageseinrichtungen zur Mehrsprachigkeit. Zeitschrift für Erziehungswissenschaft, 20(1), 237-258. https://doi.org/10.1007/s11618-017-0741-7.

Kratzmann, J., Smidt, W., Pohlmann-Rother, S., \& Kuger, S. (2013). Interkulturelle Orientierungen und pädagogische Prozesse im Kindergarten. In G. Faust (Hrsg.), Einschulung. Ergebnisse aus der Studie „Bildungsprozesse, Kompetenzentwicklung und Selektionsentscheidungen im Vorschul- und Schulalter (BiKS)“ (1. Aufl., S. 97-110). Münster: Waxmann.

Kuger, S., \& Kluczniok, K. (2009). Prozessqualität im Kindergarten - Konzept, Umsetzung und Befunde. In H.-G. Roßbach \& H.-P. Blossfeld (Hrsg.), Frühpädagogische Förderung in Institutionen (Zeitschrift für Erziehungswissenschaft: Sonderheft 11, S. 159-178). Wiesbaden: VS. https://doi.org/10. 1007/978-3-531-91452-7_11.

Kunter, M., \& Trautwein, U. (2013). Psychologie des Unterrichts (StandardWissen Lehramt, Bd. 3895). Paderborn: Ferdinand Schöningh.

Lane, J. (2008). Young children and racial justice. Taking action for racial equality in the early years - understanding the past, thinking about the present, planning for the future. London: National Children's Bureau.

Lehrl, S., Kuger, S., \& Anders, Y. (2014). Soziale Disparitäten beim Zugang zu Kindergartenqualität und differenzielle Konsequenzen für die vorschulische mathematische Entwicklung. Unterrichtswissenschaft, 42, 132-151.

Linberg, T., \& Wenz, S.E. (2017). Ausmaß und Verteilung sozioökonomischer und migrationsspezifischer Ungleichheiten im Sprachstand fünfjähriger Kindergartenkinder. Journal for educational research online, 9, 77-98.

MacNaughton, G., \& Davis, K. (2009). "Race” and early childhood education. An international approach to identity, politics, and pedagogy (1. Aufl., Critical cultural studies of childhood). New York: Palgrave Macmillan.

McLaughlin, B., \& McLeod, B. (1997). Lernen in einer Zweitsprache. Entwicklungen in den USA. Bildung und Erziehung, 50, 9-21.

Mischo, C., \& Fröhlich-Gildhoff, K. (2011). Professionalisierung und Professionsentwicklung im Bereich der frühen Bildung. Frühe Bildung. https://doi.org/10.1026/2191-9186/a000001.

Morgan, M. (2016). Erziehungspartnerschaft und Erziehungsdivergenzen. Die Bedeutung divergierender Konzepte von Erzieherinnen und Migranteneltern (Dissertation). Wiesbaden: Springer.

Muthén, L. K., \& Muthén, B.O. (2015). Mplus user's guide (7. Aufl.). Los Angeles: Muthén \& Muthén.

Nespor, J. (1987). The role of beliefs in the practice of teaching. Journal of Curriculum Studies, 19, 317-328. https://doi.org/10.1080/0022027870190403.

Neugebauer, U. (2010). Keine Outcomes trotz Kompetenzüberzeugung? Qualifikationen und Selbsteinschätzungen von Sprachförderkräften in KiTa's. Empirische Sonderpädagogik, 2, 34-47.

Neumann, U. (2005). Kindertagesangebote für unter sechsjährige Kinder mit Migrationshintergrund. In Sachverständigenkommission Zwölfter Kinder- und Jugendbericht (Hrsg.), Bildung, Betreuung und Erziehung von Kindern unter sechs Jahren (Bd. 1, S. 175-226). München: Verlag Deutsches Jugendinstitut.

OECD (2016). Exzellenz und Chancengerechtigkeit in der Bildung (PISA 2015 Ergebnisse, Bd. I). Paris: OECD Publishing. https://doi.org/10.1787/9789264267879-de.

Parker, K., Hannah, E., \& Topping, K. J. (2006). Collective teacher efficacy, pupil attainment and socio-economic status in primary school. Improving Schools, 9, 111-129. https://doi.org/10.1177/ 1365480206064965 . 
Petermann, F. (2015). Alltagsintegrierte Förderung oder Förderprogramme im Vorschulalter? Frühe Bildung, 4, 161-164. https://doi.org/10.1026/2191-9186/a000220.

Pettigrew, T.F. (1998). Intergroup contact theory. Annual review of psychology, 49, 65-85. https://doi.org/ 10.1146/annurev.psych.49.1.65.

Pfost, M. (2015). Children's phonological awareness as a predictor of reading and spelling. A systematic review of longitudinal research in German-speaking countries. Zeitschrift für Entwicklungspsychologie und Pädagogische Psychologie, 47, 123-138. https://doi.org/10.1026/0049-8637/a000141.

Pianta, R., Howes, C., Burchinal, M., Bryant, D., Clifford, R., \& Early, D. (2005). Features of prekindergarten programs, classrooms, and teachers: do they predict observed classroom quality and child-teacher interactions? Applied Developmental Science, 9, 144-159. https://doi.org/10.1207/ s1532480xads0903_2.

Piske, T. (2014). Empirische Befunde zur Rolle des Alters beim Zweitspracherwerb. In H. Böttger \& G. Gien (Hrsg.), The multilingual brain. Zum neurodidaktischen Umgang mit Mehrsprachigkeit (Eichstätter Schriftenreihe zum Kontinuum des Lernens, Bd. 9, S. 20-32). Berlin: epubli.

Relikowski, I., Schneider, T., \& Linberg, T. (2015). Rezeptive Wortschatz- und Grammatikkompetenzen von Fünfjährigen mit und ohne Migrationshintergrund. Frühe Bildung, 4, 135-143. https://doi.org/ $10.1026 / 2191-9186 / \mathrm{a} 000218$.

Ross, J., \& Bruce, C. (2007). Professional development effects on teacher efficacy: results of randomized field trial. The Journal of Educational Research, 101, 50-60. https://doi.org/10.3200/JOER.101.1.5060.

Roßbach, H.-G., Anders, Y., \& Tietze, W. (2016). Wissenschaftliche Evaluation des Bundesprogramms „Schwerpunkt-Kitas Sprache \& Integration“ - Abschlussbericht. Bamberg/Berlin: Otto-FriedrichUniversität Bamberg/Freie Universität Berlin.

Roßbach, H.-G., Kluczniok, K., \& Kuger, S. (2009). Auswirkungen eines Kindergartenbesuchs auf den kognitiv-leistungsbezogenen Entwicklungsstand von Kindern. Ein Forschungsüberblick. In H.G. Roßbach \& H.-P. Blossfeld (Hrsg.), Frühpädagogische Förderung in Institutionen (Zeitschrift für Erziehungswissenschaft: Sonderheft 11, S. 139-158). Wiesbaden: VS. https://doi.org/10.1007/9783-531-91452-7_10.

Sachse, S. (2001). Ein Sprachförderprogramm für Vorschulkinder. Evaluation und entwicklungspsychologische Fundierung. Saarbrücken: VDM Verlag Dr. Müller.

Sachse, S., Budde, N., Rinker, T., \& Groth, K. (2012). Evaluation einer Sprachfördermaßnahme für Vorschulkinder. Frühe Bildung, 1, 194-201. https://doi.org/10.1026/2191-9186/a000062.

Sachverständigenrat Deutscher Stiftungen für Integration und Migration (2013). Hürdenlauf zur Kita. Warum Eltern mit Migrationshintergrund ihr Kind seltener in die frühkindliche Tagesbetreuung schicken. Berlin: Eigenverlag.

Schlösser, E. (2012). Zusammenarbeit mit Eltern - interkulturell. Informationen und Methoden zur Kooperation mit deutschen und zugewanderten Eltern in Kindergarten, Grundschule und Familienbildung (3. Aufl., Pädagogische Kompetenz, Bd. 1). Münster: Ökotopia.

Schröder, M. B. (2012). Effekte professioneller Sprachförderung in Kindertagesstätten. Eine experimentell kontrollierte Intervention zur Förderung von Deutsch als Zweitsprache bei Kindern mit Migrationshintergrund. Potsdam: Univ.

Schroeter, R. (2014). Eine Bestandsaufnahme von Überzeugungen (beliefs) Lehramtsstudierender zu Lehrerbildung und Lehrerberuf (Dissertation). Leipzig: Leipziger Universitätsverlag.

Schwarzer, R., \& Jerusalem, M. (2002). Das Konzept der Selbstwirksamkeit. In M. Jerusalem \& D. Hopf (Hrsg.), Selbstwirksamkeit und Motivationsprozesse in Bildungsinstitutionen (Zeitschrift für Pädagogik: Beiheft 44, S. 28-53). Weinheim: Beltz.

Schweitzer, F., Biesinger, A., Blaicher, H.-P., Edelbrock, A., Haußmann, W., \& Ilg, W. (2011). Interreligiöse und interkulturelle Bildung in Kindertagesstätten - Befunde aus der Erzieherinnenbefragung. In F. Schweitzer, A. Edelbrock \& A. Biesinger (Hrsg.), Interreligiöse und interkulturelle Bildung in der Kita. Eine Repräsentativbefragung von Erzieherinnen in Deutschland - interdisziplinäre, interreligiöse und internationale Perspektiven (Interreligiöse und interkulturelle Bildung im Kindesalter Bd. 3, S. 29-54). Münster: Waxmann.

Schwippert, K., Wendt, H., \& Tarelli, I. (2012). Kapitel VII Lesekompetenzen von Schülerinnen und Schülern mit Migrationshintergrund. In W. Bos, I. Tarelli, A. Bremerich-Vos \& K. Schwippert (Hrsg.), IGLU 2011. Lesekompetenzen von Grundschulkindern in Deutschland im internationalen Vergleich (S. 191-207). Münster: Waxmann.

Settlage, J., Southerland, S. A., Smith, L. K., \& Ceglie, R. (2009). Constructing a doubt-free teaching self: self-efficacy, teacher identity, and science instruction within diverse settings. Journal of Research in Science Teaching, 46, 102-125. https://doi.org/10.1002/tea.20268. 
Simon, S., \& Sachse, S. (2013). Anregung der Sprachentwicklung durch ein Interaktionstraining für Erzieherinnen. Diskurs Kindheits- und Jugendforschung, 8, 379-397.

Siwatu, K. O. (2007). Preservice teachers' culturally responsive teaching self-efficacy and outcome expectancy beliefs. Teaching and Teacher Education, 23, 1086-1101. https://doi.org/10.1016/j.tate.2006. 07.011.

Siwatu, K. O., Chesnut, S.R., Alejandro, A. Y., \& Young, H. A. (2016). Examining preservice teachers' culturally responsive teaching self-efficacy doubts. The Teacher Educator, 51, 277-296. https://doi. org/10.1080/08878730.2016.1192709.

Spiess, C. K., Büchel, F., \& Wagner, G. G. (2003). Children's school placement in Germany: does kindergarten attendance matter? Early childhood research quarterly, 18, 255-270. https://doi.org/10.1016/ S0885-2006(03)00023-1.

Stitzinger, U., \& Lüdtke, U. (2014). Mehrsprachigkeit als Potenzial in KiTa-Teams (Nifbe-Beiträge zur Professionalisierung, Bd. 2). Osnabrück: Nifbe.

Sulzer, A. (2013). Kulturelle Heterogenität in Kitas. Anforderungen an Fachkräfte; eine Expertise der Weiterbildungsinitiative Frühpädagogische Fachkräfte (WiFF). München: DJI.

Tietze, W., \& Meischner, T. (1998). Wie gut sind unsere Kindergärten? Eine Untersuchung zur pädagogischen Qualität in deutschen Kindergärten. Neuwied: Luchterhand.

Tietze, W., Becker-Stoll, F., Bensel, J., Eckhardt, A. G., Haug-Schnabel, G., \& Kalicki, B. (2013). NUBBEK - Nationale Untersuchung zur Bildung, Betreuung und Erziehung in der frühen Kindheit. Fragestellungen und Ergebnisse im Überblick. Weimar: verlag das netz.

Tschannen-Moran, M., \& Hoy, A.W. (2001). Teacher efficacy: capturing an elusive construct. Teaching and Teacher Education, 17, 783-805. https://doi.org/10.1016/S0742-051X(01)00036-1.

Van Dick, R., Wagner, U., Adams, C., \& Petzel, T. (1997). Einstellungen zur Akkulturation: Erste Evaluation eines Fragebogens an sechs deutschen Stichproben. Gruppendynamik, 28, 83-92.

Van de Vijver, F. J.R., Breugelmans, S. M., \& Schalk-Soekar, S. R. G. (2008). Multiculturalism: construct validity and stability. International Journal of Intercultural Relations, 32, 93-104. https://doi.org/10. 1016/j.ijintrel.2007.11.001.

Viernickel, S., Nentwig-Gesemann, I., Nicolai, K., Schwarz, S., \& Zenker, L. (2013). Schlüssel zu guter Bildung, Erziehung und Betreuung - Bildungsaufgaben, Zeitkontingente und strukturelle Rahmenbedingungen in Kindertageseinrichtungen. Forschungsbericht. http://docplayer.org/17635320Forschungsbericht-schluessel-zu-guter-bildung-erziehung-und-betreuung.html. Zugegriffen: 21 . Aug. 2016.

Vorauer, J. D., Gagnon, A., \& Sasaki, S. J. (2009). Salient intergroup ideology and intergroup interaction. Psychological science, 20, 838-845. https://doi.org/10.1111/j.1467-9280.2009.02369.x.

Wagner, U., Christ, O., Pettigrew, T.F., Stellmacher, J., \& Wolf, C. (2006). Prejudice and minority proportion: contact instead of threat effects. Social Psychology Quarterly, 69, 380-390. https://doi.org/10. 1177/019027250606900406.

Weins, C. (2011). Gruppenbedrohung oder Kontakt? Kölner Zeitschrift für Soziologie und Sozialpsychologie, 63, 481-499. https://doi.org/10.1007/s11577-011-0141-6.

Welch-Sing, E., \& Pagonis, G. (2011). Vergleichsstudie zur Wirksamkeit zweier Sprachförderansätze: „Deutsch für den Schulstart“ und „Denkendorfer Modell“ (Abschlussbericht).

Wieduwilt, N., Lehrl, S., \& Anders, Y. (submitted). Preschool teachers' pedagogical beliefs in the field of language and literacy education. Teaching and Teacher Education.

Wischmeier, I. (2012). „Teachers’ Beliefs“: Überzeugungen von (Grundschul-) Lehrkräften über Schüler und Schülerinnen mit Migrationshintergrund - Theoretische Konzeption und empirische Überprüfung. In W. Wiater \& D. Manschke (Hrsg.), Verstehen und Kultur (S. 167-189). Wiesbaden: VS. https://doi.org/10.1007/978-3-531-94085-4_8.

Wolf, K. M., Felbrich, A., Stanat, P., \& Wendt, W. (2011). Evaluation der kompensatorischen Sprachförderung in Brandenburger Kindertagesstätten. Empirische Pädagogik, 25, 423-438.

Wolsko, C., Park, B., \& Judd, C. M. (2006). Considering the Tower of Babel: correlates of assimilation and Multiculturalism among ethnic minority and majority groups in the United States. Social Justice Research, 19, 277-306. https://doi.org/10.1007/s11211-006-0014-8.

Zee, M., \& Koomen, H. M. Y. (2016). Teacher self-efficacy and its effects on classroom processes, student academic adjustment, and teacher well-being. Review of Educational Research, 86, 981-1015. https:// doi.org/10.3102/0034654315626801. 